\title{
Uniform rectifiability and singular sets
}

\author{
by \\ Guy DAVID \\ Université Paris-Sud, Mathématiques, \\ Bâtiment 425, 91405 Orsay Cedex. \\ and

\section{Stephen SEMMES} \\ Mathematics Rice University, P.O. Box 1892, Houston, Texas 77251, U.S.A. \\ et Institut des Hautes Études Scientifiques, \\ 35, route de Chartres, 91440, Bures-sur-Yvette, France.
}

\begin{abstract}
Under what conditions can one say something about the geometric structure of the singular set of a function? A famous result of this type states that the singular set (= set of non-Lebesgue points) of a function of bounded variation on $\mathbf{R}^{n}$ is (countably) rectifiable. In this paper we shall be concerned with quantitative forms of rectifiability, and we give a quantitative version of this theorem. We also give uniform rectifiability results for the singular sets of minimizers of higher-dimensional versions of the Mumford-Shah functional. Along the way we shall encounter some generalizations of the usual topological notion of a set "separating" points in the complement, including one which is based on the failure of Poincaré inequalities on the complement of the given set.
\end{abstract}

RÉsumé. - Que peut-on dire de la structure géométrique de l'ensemble de singularités d'une fonction $f$ définie sur $\mathbf{R}^{n}$ ? Un théorème classique dit que si $f$ est à variation bornée, alors l'ensemble singulier de $f$ (c'està-dire le complémentaire de l'ensemble des points de Lebesgue de $f$ ) est dénombrablement rectifiable. Dans cet article, on donne une version quantifiée de ce théorème utilisant la notion de rectifiabilité uniforme.

The first author is supported by the U.S. National Science Foundation. 
On donne également un résultat de rectifiabilité uniforme des ensembles singuliers associés aux sections minimisantes pour la fonctionnelle de Mumford-Shah en dimension quelconque. L'une des idées directrices de l'article est que la rectifiabilité d'un ensemble de codimension 1 est en rapport étroit avec la manière dont il sépare localement $\mathbf{R}^{n}$ en composantes. On y trouve en particulier une notion de séparation basée sur l'absence de bonnes inégalités de Poincaré dans le complémentaire.

\section{INTRODUCTION}

In this paper we shall be concerned with quantitative rectifiability properties of sets in $\mathbf{R}^{n}$ with Hausdorff dimension equal to $n-1$, especially sets which arise as the collection of singularities of some function. We shall review the necessary background information in the next section - the definition of "uniform rectifiability" in particular - but for the moment let us proceed with a vague description of our goals and intentions.

Codimension 1 is special in the study of rectifiable sets, because rectifiability properties of a set $E$ in $\mathbf{R}^{n}$ can often be detected by the extent to which $E$ separates points in $\mathbf{R}^{n} \backslash E$. (For the record: two points in the complement of $E$ are separated by $E$ if they lie in different components of the complement.) The idea is that if $E$ has locally finite $(n-1)$-dimensional Hausdorff measure, say, and if $E$ were not rectifiable, then it would be too scattered in some places to separate points properly. (Self-similar Cantor sets provide good examples of sets which are unrectifiable and too scattered to separate any pair of points in the complement.) Thus conditions which say that $E$ does a good job of separating points in its complement in $\mathbf{R}^{n}$, together with bounds on the $(n-1)$-dimensional Hausdorff measure of $E$, ought to imply rectifiability properties of $E$.

In the context of classical rectifiability such results are well known and easy to derive from famous theorems of Besicovitch and Federer (namely, the fact that the projection of a totally unrectifiable set onto almost any hyperplane has measure zero). In the context of uniform rectifiability we do not have such powerful general tools available, but there are still reasonable results of this type. (See Section 2.)

In this paper we shall deal with different notions of separation. Instead of taking two points in the complement of $E$ and asking whether they lie in different complementary components, so that there is no curve that joins 
the two points without touching $E$, we shall ask whether it is difficult to get from one point to the other without touching $E$. For instance, this could mean that there is not a nice big family of curves joining one point to the other in $\mathbf{R}^{n} \backslash E$. Actually, it will be convenient to formulate the existence of such pairs of points in dual terms, as the failure of suitable Poincare inequalities on $\mathbf{R}^{n} \backslash E$.

We shall prove in Section 3 a criterion for uniform rectifiability of a set $E$ in $\mathbf{R}^{n}$ in terms of the uniform failure of Poincare inequalities at most scales and locations in $\mathbf{R}^{n} \backslash E$. We use this criterion in Section 5 to obtain uniform rectifiability results for the the singular sets of minimizers of a family of variants of the Mumford-Shah functional in $\mathbf{R}^{n}$ (which are described at the beginning of Section 5). For the original Mumford-Shah functional in $\mathbf{R}^{2}$ this gives an alternate proof of the main result in [DS4]. The new proof has the advantage that it works in higher dimensions.

In Section 4 we give a uniformized version of the theorem that the singular set of a function of bounded variation is rectifiable. (See conclusion (16) of Theorem 4.5 .9 on p. 483 of [Fe] and Chapter 4 of [Gi] for the classical result.) In the uniformized version we replace the assumption of bounded variation with a Carleson measure condition on the distributional gradient of the function, and we require a uniform bound on the size of the jump discontinuities. Conversely, we prove that any uniformly rectifiable set (of codimension 1) arises as the singular set of a function with the same properties. See Section 4 for details.

In Section 6 we mention a different variational problem whose minimizers are rectifiable sets with prescribed but generalized mean curvature. There is a natural potential criterion for uniform rectifiability, but we produce counterexamples to show that it is very wrong. By contrast, there are natural regularity theorems under stronger conditions on the mean curvature which are analogous to the usual results for minimal surfaces in codimension 1.

Note that Sections 2, 3, and 5 are independent of Section 4 and can be read by themselves. Section 4 is logically independent of Section 3, but it involves similar ideas and makes many references to analogous points in Section 3.

\section{A REVIEW OF UNIFORM RECTIFIABILITY}

For the rest of this paper $E$ will denote a $d$-dimensional (Ahlfors) regular set in $\mathbf{R}^{n}$. (Eventually $n$ will be $d+1$.) This means that $E$ is closed and that there exists a nonnegative Borel measure $\mu$ with support equal to $E$ 
and a constant $C_{0} \geq 1$ such that

$$
C_{0}^{-1} r^{d} \leq \mu(E \cap B(x, r)) \leq C_{0} r^{d}
$$

for all $x \in E$ and $r>0$. Here (and forever) $B(x, r)$ denotes the open ball with center $x$ and radius $r$.

For example, a $d$-plane is regular, with $\mu$ taken to be Hausdorff measure on the plane. In general, a regular set has the same kind of mass distribution as a $d$-plane, but it could be very different geometrically. There are selfsimilar Cantor sets, snowflake curves, tree-like fractals, etc., which are regular. (For the snowflakes and trees one must have $d>1$, but there are Cantor sets with arbitrary positive dimension.)

It is easy to see that if $\mu$ is as above, then $\mu$ must be equivalent in size to the restriction to $E$ of $d$-dimensional Hausdorff measure, which we denote by $H^{d}$. Thus we may as well assume that $\mu$ is $\left.H^{d}\right|_{E}$, but this will not help us.

Recall that a set $A$ in $\mathbf{R}^{n}$ is said to be rectifiable with dimension $d$, $d \in \mathbf{Z}_{+}$, if there is a set $A_{0} \subseteq A$ such that $H^{d}\left(A \backslash A_{0}\right)=0$ and $A_{0}$ is contained in a countable union of $d$-dimensional $C^{1}$ submanifolds of $\mathbf{R}^{n}$, or, equivalently, a countable union of Lipschitz images of $\mathbf{R}^{d}$. (In the basic reference [Fe], however, this is called "countable rectifiability".) We shall not really need this definition here, but it is helpful to recall it before stating the definition of uniform rectifiability. One of the main points is that classical rectifiability says that a set behaves well asymptotically at almost all of its points, but without controlling the behavior at any definite scale. Uniform rectifiability is designed to make up for this deficiency. We define it first for the $d=1$ case, which is simpler.

Definition 2.2. - Let $E$ be a 1-dimensional regular set in $\mathbf{R}^{n}$. We say that $E$ is uniformly rectifiable if there is a regular curve $\Gamma$ that contains $E$.

Recall that a regular curve is a set $\Gamma \subseteq \mathbf{R}^{n}$ of the form $\Gamma=z(\mathbf{R})$, where $z: \mathbf{R} \rightarrow \mathbf{R}^{n}$ is Lipschitz (so that

$$
|z(s)-z(t)| \leq C|s-t|
$$

for some $C>0$ and all $s, t \in \mathbf{R}$ ) and satisfies

$$
|\{s \in \mathbf{R}: z(s) \in B(y, r)\}| \leq C r \quad \text { for all } y \in \mathbf{R}^{n} \text { and } r>0
$$

(and for some other $C>0$ ). Regular curves are allowed to cross themselves, but (2.4) limits the extent of this crossing. 
Regular curves are almost the same as connected 1-dimensional regular sets. Regular curves are clearly connected regular sets, but conversely any connected 1-dimensional regular set in $\mathbf{R}^{n}$ is contained in a regular curve. This is not very hard to prove. (Theorem 1.8 on p. 6 of [DS3] is helpful in this regard.)

The idea of uniform rectifiability is that it prevents the set from ever being too scattered (like a Cantor set) by requiring the existence of a reasonable parameterization by a Euclidean space. In the higher-dimensional case, among the several equivalent definitions of uniform rectifiability there is unfortunately not one which is so much simpler than the others. We shall use here the one that is closest to Definition 2.2. (See [DS3] for some alternatives.)

Let $A_{1}\left(\mathbf{R}^{d}\right)$ denote the Muckenhoupt class of $A_{1}$ weights on $\mathbf{R}^{d}$, that is, the class of positive measurable functions $\omega(x)$ on $\mathbf{R}^{d}$ such that

$$
\rho^{-d} \int_{B(x, \rho)} \omega(y) d y \leq C \underset{y \in B(x, \rho)}{\operatorname{essinf}} \omega(y)
$$

for some $C>0$ and all $x \in \mathbf{R}^{d}$ and $\rho>0$. This condition may appear to be a little mysterious, but it means, roughly, that $\omega$ does not oscillate too wildly, on average, and that $\omega$ never gets too small too quickly. The simplest example is $\omega \equiv 1$. One of the basic results about $A_{1}$ weights is that if $\omega \in A_{1}$, then there is a $q>1$ and a $C^{\prime}>0$ so that

$$
\left(\rho^{-d} \int_{B(x, \rho)} \omega(y)^{q} d y\right)^{\frac{1}{q}} \leq C^{\prime} \rho^{-d} \int_{B(x, \rho)} \omega(y) d y
$$

for all $x \in \mathbf{R}^{d}$ and $\rho>0$. This makes precise the idea that $\omega$ cannot get too big, on average.

If $\omega \in A_{1}\left(\mathbf{R}^{d}\right.$ ), then a mapping $z: \mathbf{R}^{d} \rightarrow \mathbf{R}^{m}$ (for some $m \geq d$ ) is said to be $\omega$-regular if there is a $C>0$ such that

$$
|z(x)-z(y)| \leq C\left\{\int_{B\left(\frac{x+y}{2},|y-x|\right)} \omega(u) d u\right\}^{\frac{1}{d}}
$$

for all $x, y \in \mathbf{R}^{d}$ and

$$
\omega\left\{x \in \mathbf{R}^{d}: z(x) \in B(y, r)\right\} \leq C r^{d}
$$

for all $y \in \mathbf{R}^{m}$ and $r>0$. Here $\omega(A)$ denotes the $\omega(x) d x$ measure of $A$, $A \subseteq \mathbf{R}^{d}$. This is of course analogous to the definition of regular curves Vol. 13, n 4-1996. 
above, and indeed (2.7) and (2.8) are equivalent to (2.3) and (2.4) when $\omega \equiv 1$ and $d=1$. In general one can think of $\omega$ as representing a controlled perturbation of the geometry of $\mathbf{R}^{d}$. The point of allowing $\omega$ here is to make it easier to produce regular mappings and thereby ameliorate the difficulty of building $d$-dimensional parameterizations. When $d=1$ this is not an issue, because it is then much easier to build parameterizations, and in any case we could reduce to the $\omega \equiv 1$ case by making an easy change of variables on the real line.

Definition 2.9. - A set $E$ in $\mathbf{R}^{n}$ is uniformly rectifiable (with dimension $d$ ) if it is a $d$-dimensional regular set and if there is a weight $\omega \in A^{1}\left(\mathbf{R}^{d}\right)$ and an $\omega$-regular mapping $z: \mathbf{R}^{d} \rightarrow \mathbf{R}^{n+1}$ such that $E \subseteq z\left(\mathbf{R}^{d}\right)$.

In this definition we are identifying $\mathbf{R}^{n}$ with a subset of $\mathbf{R}^{n+1}$ in the obvious way. We need to allow $z$ to take values in $\mathbf{R}^{n+1}$ (instead of $\mathbf{R}^{n}$ ) in order to make it easier to find parameterizations that do not cross themselves too often. When $n \geq 2 d$ this problem can be avoided and we can replace $n+1$ by $n$.

There are many alternative characterizations of uniform rectifiability, in purely geometric terms and also in terms of analysis. Some of these alternatives are given in terms of uniformly bilipschitz parameterizations of large pieces of the set. (That is, we can get rid of the $\omega$ if we are willing to not parameterize the whole set at once.) See [DS3] for an extensive discussion of the various characterizations. The definition above is good for making precise the idea that a uniformly rectifiable set is one that can be rather well parameterized by $\mathbf{R}^{d}$, but it is not so easy to verify. In Definition 2.12 below we give a more convenient criterion, but first we need an auxiliary notion.

Definition 2.10. - Let $\mathcal{B}$ be a subset of $E \times \mathbf{R}_{+}$. We say that $\mathcal{B}$ is a Carleson set if there is a constant $C$ such that

$$
\int_{0}^{r} \int_{E \cap B(x, r)} \mathbf{1}_{\mathcal{B}}(y, t) \frac{d \mu(y) d t}{t} \leq C r^{d}
$$

for all $x \in E$ and $r>0$.

Here the measure $\mu$ is the same as the one in (2.1), and it can be taken to be $\left.H^{d}\right|_{E}$. The measure $\frac{d \mu(y) d t}{t}$ scales like a $d$-dimensional measure, even though it lives on a $d+1$-dimensional space, and it can give infinite mass to bounded sets (like the product of a ball with $(0,1)$ ). Roughly speaking, a Carleson set is a subset of $E \times \mathbf{R}_{+}$which behaves in terms of its mass distribution as though it were $d$-dimensional, at least from the perspective of $E \times\{0\}$. In particular Carleson sets are very small compared to $E \times \mathbf{R}_{+}$. 
For the sake of convenience let us agree to apply the notion of Carleson sets even to nonmeasurable sets $\mathcal{B}$, in which case we should use outer measures in (2.11). This is not a serious issue; in most cases there will be a kind of discreteness present which makes the relevant Carleson condition equivalent to a statement about sums. (For instance, the measurability is pleasantly irrelevant in the proof of Sublemma 3.9.) The main point is that the reader should not waste time worrying about measurability when it is not really necessary.

A compact subset of $E \times \mathbf{R}_{+}$is always a Carleson set. Some noncompact examples of Carleson sets are $\mathcal{B}=\left\{(x, t) \in E \times \mathbf{R}_{+}: t_{0}<t<1000 t_{0}\right\}$ (for any given $t_{0}>0$ ) and $\mathcal{B}=\left\{(x, t) \in E \times \mathbf{R}_{+}:\left|x-x_{0}\right| \leq 1000 t\right\}$ (for any given $x_{0} \in E$ ). It is not difficult to check that these really are Carleson sets, with a constant $C$ which is bounded independently of $t_{0}$ and $x_{0}$. A more interesting class of examples is given as follows. Let $J$ be a set of integers, and set $\mathcal{B}=\left\{(x, t) \in E \times \mathbf{R}_{+}: 2^{j} \leq t \leq 2^{j+1}\right.$ for some $\left.j \in J\right\}$. This is a Carleson set if and only if $J$ is a finite set. In general Carleson sets do not need to be so neatly layered, but this example does provide a reasonable illustration of how large a Carleson set can be.

Here is another amusing example. Suppose that $E$ is a $d$ plane, and let $Q$ be a $(d-1)$-plane contained in $E$. Then $\mathcal{B}=$ $\left\{(x, t) \in E \times \mathbf{R}_{+}: \operatorname{dist}(x, Q)<t\right\}$ is a Carleson set. The assumption that $E$ and $Q$ are planes is not crucial; there are very general versions of this example. The point is that a Carleson set can have infinitely many layers, but only over a relatively thin subset of $E$.

In practice we shall apply the concept of Carleson sets to "bad" sets in $E \times \mathbf{R}_{+}$. That is to say, we shall have a set $\mathcal{B}$ of places in $E \times \mathbf{R}_{+}$where something bad happens, and we shall want to know that there are not too many of these bad places. The Carleson condition is often the right way to say that these bad sets are small enough that they do not cause too much trouble. The next definition provides an example of this.

Definition 2.12. - Let $E$ be a $d$-dimensional regular set in $\mathbf{R}^{n}$. We say that $E$ is locally symmetric if the following set $\mathcal{B}(\epsilon)$ is a Carleson set for each $\epsilon>0$ :

$$
\begin{aligned}
\mathcal{B}(\epsilon)=\{( & x, t) \in E \times \mathbf{R}_{+}: \text {there exist points } w, z \in E \cap B(x, t) \\
& \text { such that } \operatorname{dist}(2 z-w, E)>\epsilon t\}
\end{aligned}
$$

In other words, $E$ is locally symmetric if it is regular and if for most $(x, t) \in E \times \mathbf{R}_{+}$we have that $E$ is approximately symmetric (at the scale of $t$ ) about each of its elements in $B(x, t)$. More precisely, $\mathcal{B}(\epsilon)$ is the 
set of $(x, t) \in E \times \mathbf{R}_{+}$for which this approximate symmetry fails, and the definition of local symmetry requires that this bad set be a Carleson set, and hence small.

Clearly a $d$-plane is locally symmetric, because $\mathcal{B}(\epsilon)$ is then empty for all $\epsilon>0$. A half-plane is locally symmetric too; in this case $\mathcal{B}(\epsilon)$ is not empty, but it is still small enough to be a Carleson set. If $E$ is a smooth embedded $d$-dimensional submanifold in $\mathbf{R}^{n}$ which is also "smooth at infinity", then $E$ also satisfies the local symmetry condition. In this case $\mathcal{B}(\epsilon)$ is a compact subset of $E \times \mathbf{R}_{+}$for all $\epsilon>0$. The point is that, under these assumptions, $E$ is very well approximated by a $d$-plane in all sufficiently small balls, as well as all balls which are sufficiently large or sufficiently far out (towards infinity), and so it is certainly approximately symmetric in these balls.

Notice that we do not impose any requirements in Definition 2.12 on the way that the Carleson constants for $\mathcal{B}(\epsilon)$ depend on $\epsilon$. This is one of the reasons that the local symmetry condition is often relatively easy to check. As a practical matter, only one small choice of $\epsilon$ is ever needed in any particular application; this choice typically depends only on parameters like the relevant dimensions and the regularity constants but is not explicit.

The local symmetry condition is equivalent to asking that $E \cap B(x, t)$ be well approximated, for most $(x, t) \in E \times \mathbf{R}_{+}$, by $P \cap B(x, t)$ for some $d$-plane $P$ which depends on $(x, t)$. As always, "most $(x, t) \in E \times \mathbf{R}_{+}$" means that the exceptional set is a Carleson set in $E \times \mathbf{R}_{+}$. The precise formulation of this condition of approximation by $d$-planes is called the Bilateral Weak Geometric Lemma (BWGL) and is given in Definition 2.2 on p. 32 in [DS3]. It is easy to show that the BWGL implies the local symmetry condition, because $d$-planes are symmetric about each of their points, but it turns out that the converse is true too. This is not hard to prove, but one must use also the mass bounds on $E$ that come from Ahlfors regularity. See p. 27-30 in [DS1].

Although the local symmetry condition and the BWGL are equivalent, the local symmetry condition tends to be easier to check, and the BWGL is more convenient for deriving information.

THEOREM 2.14. - Let $E$ be a d-dimensional regular set in $\mathbf{R}^{n}$. Then $E$ is uniformly rectifiable if and only if it is locally symmetric.

This is proved in [DS3]. (See Definition 1.79 on p. 29 and Corollary 2.10 on p. 33 of [DS3]. Notice that [DS3] uses a different definition of uniform rectifiability, but the equivalence of the definitions is proved in [DS1] and is stated in Theorem 1.57 on p. 22 of [DS3].) Let us say a few words about the argument for the "if" part, which is the part that we shall need here. We already mentioned that the local symmetry condition is equivalent to 
the Bilateral Weak Geometric Lemma (BWGL). When $d=1$ we proceed as follows: the BWGL implies the Weak Connectedness Condition (WCC) trivially (see Definition 2.12 on p. 34 of [DS3]), and we can derive uniform rectifiability from the WCC using the argument on p. 69-76 of [DS3]. In general one should use the more complicated argument on p. 97-119 in [DS3]. Actually, we shall be concerned only with the codimension 1 $(d=n-1)$ case in this paper, and for this case the argument in [DS3] is shorter and simpler (but still not as nice as when $d=1$ ). For the specific applications given here the argument can be simplified further, as in Remark 3.27 below.

Next we state another criterion for uniform rectifiabilty. This one applies only to the codimension 1 case and involves the extent to which $E$ separates points in its complement.

Definition 2.15. - Let $E$ be a $d$-dimensional regular set in $\mathbf{R}^{d+1}$. We say that $E$ satisfies Condition $B$ if there is a $C_{0}>0$ such that for each $x \in E$ and each $r>0$ we can find two balls $B_{1}$ and $B_{2}$ of radius $C_{0}{ }^{-1} r$ contained in $B(x, r)$ which do not touch $E$ and (most importantly) which lie in different connected components of $\mathbf{R}^{d+1} \backslash E$. (See Figure 1.)



Fig. 1 .

A modestly different version of this condition appeared first in [Se1] in connection with $L^{2}$-boundedness of singular integral operators on $E$. It was proved in [D] that Condition B implies uniform rectifiability, and even a slightly stronger condition ("big pieces of Lipschitz graphs"). Simpler proofs of this fact are given in [DJ] and [DS2]; see also [Se2]. In this paper we shall face generalizations of Condition B in terms of weaker forms of separation, and we shall use methods similar to the ones employed in [DS2].

Notice that a hyperplane satisfies Condition B, but that a hyperplane with an open ball removed does not. This illustrates one of the drawbacks of Condition B; it is very unstable, in the sense that we can punch out a small hole from $E$ and then get a set with only one complementary component. In 
Sections 3 and 4 below we give new criteria for uniform rectifiability which are rather similar to Condition B but which do not have this drawback.

\section{THE WNPC CONDITION}

From now on, $E$ will be a $d$-dimensional regular set in $\mathbf{R}^{d+1}$. We want to define a condition on $E$ called the WNPC (Weak No Poincaré estimates in the Complement condition). The structure of this condition will be similar to that of the local symmetry condition in Definition 2.12; we shall first define a family of bad sets in $E \times \mathbf{R}_{+}$, and then we shall ask that these bad sets be Carleson sets for certain ranges of the relevant parameters. This condition will say that $E$ tries to separate its complement at most locations and scales, and the failure of Poincaré inequalities will measure this separation.

For the record, let us recall the Poincaré inequality. It has many variants, but the following will be convenient for our purposes:

$$
r^{-2(d+1)} \int_{B} \int_{B}|f(x)-f(y)| d x d y \leq C r^{-d-1} \int_{B} r|\nabla f(u)| d u
$$

for any ball $B$ with radius $r$ in $\mathbf{R}^{d+1}$. Here $C$ is a constant which depends only on $d$, and $f$ is a smooth function on $B$. (A standard approximation argument implies that (3.1) holds under the weaker assumption that the distributional gradient of $f$ is integrable on $B$.) Recall that to prove (3.1) one can simply bound $|f(x)-f(y)|$ in terms of the integral of $|\nabla f(u)|$ along the line segment that connects $x$ to $y$, average over $x$ and $y$, and apply Fubini's theorem.

A simple consequence of (3.1) is that if $H, K \subseteq B$ are measurable and $|H|,|K| \geq \delta|B|$ for some $\delta>0$ (where $|H|$ denotes the Lebesgue measure of $H$ ), then

$$
\left|m_{H} f-m_{K} f\right| \leq C(\delta) r^{-d-1} \int_{B} r|\nabla f(u)| d u .
$$

Here $m_{H} f$ denotes the mean value of $f$ over $H$ (with respect to Lebesgue measure).

Let us now proceed to the definition of the WNPC. For each choice of $C_{0}, k>1$, and $M \geq 0$ let $\mathcal{B}\left(C_{0}, k, M\right)$ be the (bad) set of $(x, t) \in E \times \mathbf{R}_{+}$ such that

$$
\left|m_{B_{1}} f-m_{B_{2}} f\right| \leq M t^{-d} \int_{B(x, k t) \backslash E}|\nabla f(u)| d u
$$


for all choices of balls $B_{1}, B_{2}$ contained in $B(x, t) \backslash E$ with radius $\geq \frac{t}{C_{0}}$, and for all functions $f \in C^{\infty}(B(x, k t) \backslash E)$. In other words, $(x, t)$ lies in our bad set if there is some amount of control on the oscillation of a function $f \in C^{\infty}(B(x, k t) \backslash E)$ in terms of the integral of $|\nabla f|$, so that we have something like the Poincaré inequality on $B(x, k t) \backslash E$. Although one normally considers the existence of a Poincaré inequality to be good, in this case it is bad, because it means that the set $E$ is scattered or has a big hole. If $(x, t)$ does not lie in our bad set, then (3.3) can fail, and this means that $E$ approximately separates some points in $B(x, t)$.

To understand all of this note that if $E$ is a $d$-plane, then $\mathcal{B}\left(C_{0}, k, M\right)$ is always empty. The reason is that we can take $f$ to be a constant on each of the two components of $\mathbf{R}^{d+1} \backslash E$, so that the right side of (3.3) vanishes, and we can make the left side arbitrarily large by choosing $B_{1}$ and $B_{2}$ so that they lie on opposite sides of $E$, and by choosing the values of $f$ on the two sides of $E$ to be sufficiently different from each other.

The same argument shows that $\mathcal{B}\left(C_{0}, k, M\right)$ is empty for all choices of $k$ and $M$ when $E$ satisfies Condition B and $C_{0}$ is as in Definition 2.15. Conversely, if $\mathcal{B}\left(C_{0}, k, M\right)=\emptyset$ for some $C_{0}$ and all $k$ and $M$, then $E$ satisfies Condition $B$. This is not hard to check; the main point is that the failure of (3.3) for large values of $k$ and $M$ leads to a function $f$ which is locally constant on the complement of $E$ but which takes different values on a pair of balls like $B_{1}, B_{2}$ above. Thus the requirement that $\mathcal{B}\left(C_{0}, k, M\right)$ be small for suitable choices of $C_{0}, k$, and $M$ can be viewed as a generalization of Condition B.

Notice that (3.3) does hold when $B(x, t) \cap E=\emptyset$ (with $k=1$ and a value of $M$ which depends on $C_{0}$ ) by (3.2). Of course in our situation $B(x, t) \cap E$ is never empty, but it can still be tame enough to allow (3.3) to hold. For instance, if $E$ is a $d$-plane with a round hole, then $\mathcal{B}\left(C_{0}, k, M\right)$ will not be empty. If $x$ lies on the boundary of the hole and if $t$ is small enough, then $(x, t)$ will lie in $\mathcal{B}\left(C_{0}, k, M\right)$. Conversely, if $(x, t) \in \mathcal{B}\left(C_{0}, k, M\right)$, then $t$ cannot be too large compared to the size of the hole, and $x$ can't be too far away from the hole (compared to $t$ ).

One can also show that (3.3) will always hold if $E$ is sufficiently scattered (and $M$ is large enough, depending on $C_{0}$ ), e.g., if $E$ is a self-similar Cantor set. If $E$ is sufficiently scattered, then one has the same kind of estimates as (3.3) as when $E$ is empty.

The moral of this story is that (3.3) will hold if $E$ is sufficiently scattered, or if its gaps are big enough, and that the failure of (3.3) means that $E$ separates some points, at least approximately. If $\mathcal{B}\left(C_{0}, k, M\right)$ is sufficiently 
sparse, then $E$ should never be too scattered, we can hope that $E$ will have good rectifiability properties.

Definition 3.4. - Let $E$ be a $d$-dimensional regular set in $\mathbf{R}^{d+1}$. We say that $E$ satisfies the WNPC ("Weak No Poincaré inequalities in the Complement" condition) if there is a $C_{0}>1$ such that $\mathcal{B}\left(C_{0}, k, M\right)$ is a Carleson set for all values of $k>1$ and $M>0$.

THEOREM 3.5. - If E is a d-dimensional regular set in $\mathbf{R}^{d+1}$ which satisfies the WNPC, then $E$ is locally symmetric (and hence uniformly rectifiable).

The last part of Theorem 3.5 follows from Theorem 2.14, but we shall say a few words later about how uniform rectifiability can be derived from the WNPC with less machinery. (See Remark 3.27 below.)

As is customary in these situations, in order to conclude that $E$ is uniformly rectifiable we do not really need $E$ to satisfy the WNPC, but only the weaker condition that $\mathcal{B}\left(C_{0}, k, M\right)$ be a Carleson set for a single choice of $C_{0}, k$, and $M$, and where $k$ and $M$ are large enough, depending on $C_{0}, d$, and the regularity constants for $E$. (See Remark 3.43 below.)

One might wonder whether the converse to Theorem 3.5 is true, i.e., whether any uniformly rectifiable set in $\mathbf{R}^{d+1}$ with dimension $d$ should satisfy the WNPC. This is certainly not the case. Take, for instance, $E$ to be a coordinate hyperplane with holes of radius $\frac{1}{10}$ at integer lattice points. This set is sufficiently porous so that any $(x, t) \in E \times \mathbf{R}_{+}$will lie in $\mathcal{B}\left(C_{0}, k, M\right)$ when $t$ is sufficiently large, assuming also that $M$ is large enough (depending on $C_{0}$ ). Thus these sets will be too large to be Carleson sets.

Let us now prove Theorem 3.5. The argument will be much the same as for Condition B in [DS2], but there will be a couple of differences.

Let $E$ be as in the theorem, a regular set which satisfies the WNPC, and let $\epsilon>0$ be given. We want to prove that the bad set $\mathcal{B}(\epsilon)$ from (2.13) is a Carleson set.

Let $C_{0}$ be as in the definition of the WNPC, and let $k, M$, and $k_{1}$ be three large constants, to be specified later. Let us begin by dispensing with the annoying set $\mathcal{B}_{1}(\epsilon)$ of pairs $(x, t) \in \mathcal{B}(\epsilon)$ which are not too far from an element of the bad set $\mathcal{B}\left(2 C_{0}, k, M\right)$, i.e., the set of pairs $(x, t)$ which satisfy

$$
\begin{aligned}
& \text { there exists } y \in E \cap B(x, t) \text { and } s \in\left[\frac{t}{k_{1}}, t\right] \\
& \text { such that }(y, s) \in \mathcal{B}\left(2 C_{0}, k, M\right) .
\end{aligned}
$$

Lemma 3.7. - $\mathcal{B}_{1}(\epsilon)$ is a Carleson set.

To prove this we begin with the following observation. 
Sublemma 3.8. - If $(y, s) \in \mathcal{B}\left(2 C_{0}, k, M\right)$, then all the pairs $\left(y^{\prime}, s^{\prime}\right) \in$ $E \times \mathbf{R}_{+}$such that $\left|y-y^{\prime}\right|<\frac{s}{4}$ and $\frac{s}{2}<s^{\prime}<\frac{3 s}{4}$ lie in $\mathcal{B}\left(C_{0}, 3 k, 2^{d} M\right)$.

Indeed, let $(y, s)$ and $\left(y^{\prime}, s^{\prime}\right)$ be as in the sublemma, let $B_{1}$ and $B_{2}$ be two balls contained in $B\left(y^{\prime}, s^{\prime}\right) \backslash E$ with radius $\geq \frac{s^{\prime}}{C_{0}}$, and let $f$ be a function in $C^{\infty}\left(B\left(y^{\prime}, 3 k s^{\prime}\right) \backslash E\right)$. Then $B_{1}, B_{2} \subseteq B(y, s) \backslash E$ and $B(y, k s) \subseteq$ $B\left(y^{\prime}, 3 k s^{\prime}\right)$, and we can use our assumption that $(y, s) \in \mathcal{B}\left(2 C_{0}, k, M\right)$ to conclude that

$$
\begin{aligned}
\left|m_{B_{1}} f-m_{B_{2}} f\right| & \leq M s^{-d} \int_{B(y, k s) \backslash E}|\nabla f(u)| d u \\
& \leq 2^{d} M s^{\prime-d} \int_{B\left(y^{\prime}, 3 k s^{\prime}\right) \backslash E}|\nabla f(u)| d u .
\end{aligned}
$$

This proves the sublemma.

To derive Lemma 3.7 from Sublemma 3.8 we shall use a general fact which we state separately.

Sublemma 3.9. - Let $\mathcal{A}$ be a subset in $E \times \mathbf{R}_{+}$, and let $k_{1}>1$ be arbitrarily large. Define $\mathcal{A}_{0}$ and $\mathcal{A}_{1}$ by $\mathcal{A}_{0}=\left\{(x, t) \in E \times \mathbf{R}_{+}:\left(x^{\prime}, t^{\prime}\right) \in \mathcal{A}\right.$ whenever $\left|x-x^{\prime}\right|<\frac{t}{4}$ and $\left.\frac{t}{2}<t^{\prime}<\frac{3 t}{4}\right\}$ and $\mathcal{A}_{1}=\left\{(y, s) \in E \times \mathbf{R}_{+}\right.$: there exists a $(z, r) \in \mathcal{A}_{0}$ such that $|y-z|<k_{1} r$ and $\left.k_{1}{ }^{-1} r<s<k_{1} r\right\}$. (Roughly speaking, $\mathcal{A}_{0}$ is smaller than $\mathcal{A}$, and $\mathcal{A}_{1}$ is a smeared-up version of $\mathcal{A}_{0}$.) If $\mathcal{A}$ is a Carleson set, then so is $\mathcal{A}_{1}$.

Lemma 3.7 follows easily from the sublemmas. Indeed, if we take $\mathcal{A}$ to be $\mathcal{B}\left(C_{0}, 3 k, 2 M\right)$, then Sublemma 3.8 implies that $\mathcal{B}_{1}(\epsilon)$ is contained in $\mathcal{A}_{1}$, and hence is a Carleson set.

Sublemma 3.9 could be proved using a simple covering argument, but it seems easier to use Fubini's theorem. Let $\mathcal{A}$ be as above. We may as well assume that $\mathcal{A}$ is open, because we can replace it with its interior without affecting $\mathcal{A}_{0}$ or $\mathcal{A}_{1}$. Let $\chi$ and $\chi_{1}$ denote the characteristic functions of $\mathcal{A}$ and $\mathcal{A}_{1}$, respectively. Then there is a constant $C>0$ such that

$$
\chi_{1}(x, t) \leq C \int_{\frac{t}{2 k_{1}}}^{2 k_{1} t} \int_{E \cap B\left(x, 2 k_{1} t\right)} \chi(y, s) \frac{d \mu(y) d s}{s^{d+1}} .
$$

Using Fubini's theorem we get that

$$
\begin{aligned}
& \int_{0}^{R} \int_{E \cap B(Z, R)} \chi_{1}(x, t) \frac{d \mu(x) d t}{t} \\
& \quad \leq C\left(k_{1}\right) \int_{0}^{2 k_{1} R} \int_{E \cap B\left(Z, 3 k_{1} R\right)} \chi(y, s) \frac{d \mu(y) d s}{s}
\end{aligned}
$$

Vol. 13, n 4-1996. 
for any $Z \in E$ and $R>0$. This last integral is at most $C\left(k_{1}\right) R^{d}$ since $\mathcal{A}$ is assumed to be a Carleson set. This proves that $\mathcal{A}_{1}$ is also a Carleson set, as desired.

Notice that Sublemma 3.9 finesses nicely the measurability issues. Even if $\mathcal{A}$ is not measurable to begin with, we can replace it by its interior, and the Carleson set $\mathcal{A}_{1}$ that we get in the end is open (by definition) and hence measurable. Thus, although the measurability of $\mathcal{B}_{1}(\epsilon)$ is a nuisance, the proof of Lemma 3.7 allows us not to bother with it by realizing $\mathcal{B}_{1}(\epsilon)$ as a subset of a measurable Carleson set.

We are left now with the interesting part of the proof of Theorem 3.5, namely, the fact that $\mathcal{B}_{2}(\epsilon)=\mathcal{B}(\epsilon) \backslash \mathcal{B}_{1}(\epsilon)$ is a Carleson set. Our strategy will be to associate, to each pair $(x, t) \in \mathcal{B}_{2}(\epsilon)$, a measurable subset $E(x, t)$ of $E$ which is not too small and for which we can control the overlaps of the $E(x, t)$ 's. This will allow us to derive estimates on the size of $\mathcal{B}_{2}(\epsilon)$ from corresponding estimates for $E$ (namely, the regularity condition (2.1)).

Let $(x, t) \in \mathcal{B}_{2}(\epsilon)$ be given. Because $(x, t) \in \mathcal{B}(\epsilon)$, there exist points $w, z \in E \cap B(x, t)$ such that the ball $D_{0}=B(2 z-w, \epsilon t)$ does not meet $E$. (Compare with (2.13).)

We now choose $k_{1}=100 \epsilon^{-1}$ and $k=500 \epsilon^{-1}$. Set $t_{1}=\frac{\epsilon t}{100}$. Since $(x, t) \notin \mathcal{B}_{1}(\epsilon)$ we get that $\left(z, t_{1}\right)$ and $\left(w, t_{1}\right)$ do not belong to $\mathcal{B}\left(2 C_{0}, k, M\right)$. Using this property of $\left(z, t_{1}\right)$, and the fact that $5 t=k t_{1}$, we get that there is a function $f_{1} \in C^{\infty}(B(z, 5 t) \backslash E)$ and two balls $D_{1,1}$ and $D_{1,2}$ such that

$$
\int_{B(z, 5 t) \backslash E}\left|\nabla f_{1}(u)\right| d u \leq 1,
$$

$D_{1,1}$ and $D_{1,2}$ are contained in $B\left(z, t_{1}\right) \backslash E$ and have radius $\geq \frac{t_{1}}{2 C_{0}}$, and

$$
\left|m_{D_{1,1}} f_{1}-m_{D_{1,2}} f_{1}\right| \geq M t_{1}^{-d} \text {. }
$$

Let $D_{1}$ be the ball $D_{1, j}, j=1,2$, such that $\left|m_{D_{1, j}} f_{1}-m_{D_{0}} f_{1}\right|$ is larger. Then (3.11) gives

$$
\left|m_{D_{1}} f_{1}-m_{D_{0}} f_{1}\right| \geq \frac{1}{2} M t_{1}^{-d} .
$$

Since $\left(w, t_{1}\right) \notin \mathcal{B}\left(2 C_{0}, k, M\right)$, we can also find (by the same argument) a function $f_{2} \in C^{\infty}(B(w, 5 t) \backslash E)$ and a ball $D_{2}$ such that

$$
\int_{B(w, 5 t) \backslash E}\left|\nabla f_{2}(u)\right| d u \leq 1,
$$

$D_{2} \subseteq B\left(w, t_{1}\right) \backslash E, D_{2}$ has radius $\geq \frac{t_{1}}{2 C_{0}}$, and

$$
\left|m_{D_{2}} f_{2}-m_{D_{1}} f_{2}\right| \geq \frac{1}{2} M t_{1}^{-d} .
$$

(See Figure 2.) 

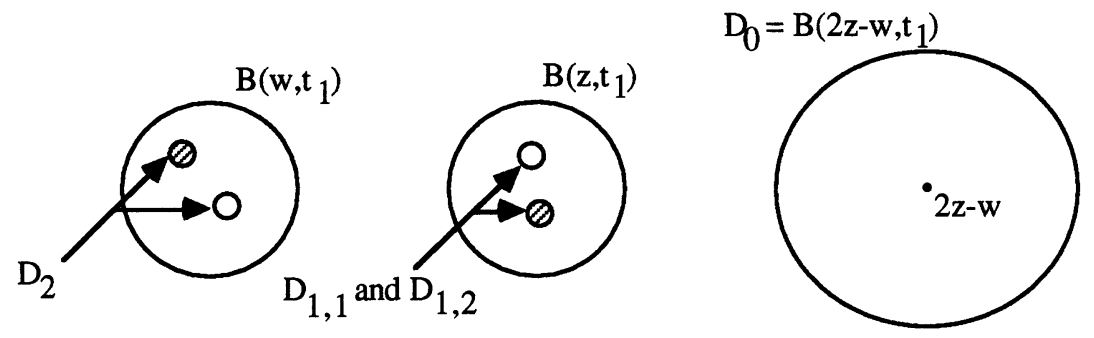

Fig. 2.

Next we want to choose a pair of balls $B_{j}=B\left(z_{j}, \frac{t_{1}}{5 C_{0}}\right), j=1,2$, with certain properties. Let $\theta(x, t)=\frac{z_{1}-z_{2}}{\left|z_{1}-z_{2}\right|}$ be the unit vector which points in the direction of the line from $z_{1}$ to $z_{2}$ (or, rather, which will point in that direction once we choose $z_{1}$ and $z_{2}$ ). We require that $2 B_{j} \subseteq D_{j}$ for $j=1,2$, and that $\theta(x, t)$ lie in a fixed finite set of unit vectors $\Theta=\Theta(\epsilon)$. It is easy to see that we can always choose these balls $B_{j}$ in this way if $\Theta$ is sufficiently dense as a subset of the unit sphere in $\mathbf{R}^{d+1}$.

Our next goal is to define the set $E(x, t)$ mentioned earlier. To do this we need some auxiliary constructions.

Set $z_{0}=2 z_{1}-z_{2}$ and $B_{0}=B\left(z_{0}, \frac{t_{1}}{5 C_{0}}\right)$. Thus $B_{0}$ is the reflection of $B_{2}$ about $z_{1}$, and we have that $2 B_{0} \subseteq D_{0}$ by definitions.

Denote by $P_{j}$ the hyperplane through $z_{j}$ which is orthogonal to $\theta(x, t)$, $j=0,1,2$. For each $\xi \in P_{1} \cap B_{1}$ let $L(\xi)$ be the line through $\xi$ determined by the direction $\theta(x, t)$. Let $L^{+}(\xi)$ be the portion of $L(\xi)$ which lies between $P_{1}$ and $P_{2}$, and let $L^{-}(\xi)$ denote the portion between $P_{0}$ and $P_{1}$. (See Figure 3.) Finally let $Z^{ \pm}$denote the set of $\xi \in P_{1} \cap B_{1}$ such that $L^{ \pm}(\xi)$ intersects $E$.

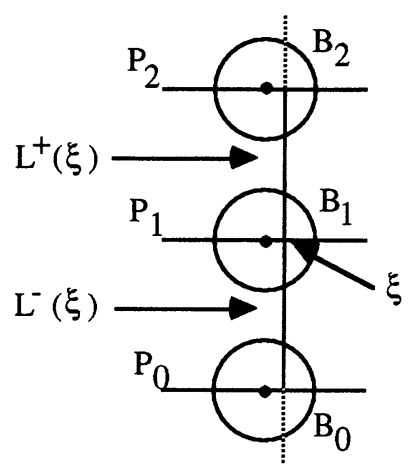

Fig. 3. 
LEMMA 3.15. - If $M$ is chosen large enough, then $H^{d}\left(Z^{+} \cap Z^{-}\right) \geq$ $\frac{1}{2} H^{d}\left(P_{1} \cap B_{1}\right) \geq C^{-1} t_{1}^{d}$.

Remember that $H^{d}$ denotes $d$-dimensional Hausdorff measure. (In the inequalities above this reduces to Lebesgue measure on $P_{1}$.) Set $\sigma=t_{1}^{-d} H^{d}\left(P_{1} \cap B_{1}\right)$, so that $C^{-1} \leq \sigma \leq C$. It clearly suffices to show that

$$
H^{d}\left(P_{1} \cap B_{1} \backslash Z^{+}\right) \leq \frac{1}{4} \sigma t_{1}^{d}
$$

and a similar estimate for $Z^{-}$. The idea is that if (3.16) were not true, then there would be many paths that join $B_{1}$ to $B_{2}$, and then the difference between the mean values of $f_{2}$ on these balls would not be too large, which would contradict (3.14). Let us make this precise.

Suppose that (3.16) does not hold, and set

$$
F=\left\{\xi \in P_{1} \cap B_{1} \backslash Z^{+}: \int_{L(\xi) \cap B(w, 5 t) \backslash E}\left|\nabla f_{2}\right| d H^{1} \leq \frac{8}{\sigma t_{1}^{d}}\right\} .
$$

Because of (3.13) and the definition of $F$, we obtain from Fubini and Tchebytchev that $H^{d}\left(P_{1} \cap B_{1} \backslash\left(Z^{+} \cup F\right)\right) \leq \frac{\sigma t_{1}^{d}}{8}$, and so

$$
H^{d}(F) \geq \frac{\sigma t_{1}^{d}}{8}
$$

since we are assuming that (3.16) is false.

Next let $R_{j}, j=1,2$, denote the set of points $u$ that lie on some $L(\xi), \xi \in F$, and which satisfy $\operatorname{dist}\left(u, P_{j}\right) \leq \frac{t_{1}}{5 C_{0}}$. Notice that $R_{j} \subseteq 2 B_{j} \subseteq D_{j} \subseteq B(w, 5 t) \backslash E$, and also that $H^{d+1}\left(R_{j}\right) \geq C^{-1} t_{1}^{d+1}$ because of (3.18). Denote by $m_{j}$ the mean value of $f_{2}$ on the set $R_{j}$. Then

$$
\left|m_{j}-m_{D_{j}} f_{2}\right| \leq C t_{1}^{-d}
$$

by (3.2) and (3.13).

Let us now estimate $\left|m_{1}-m_{2}\right|$. Notice that $m_{j}=$ $H^{d}(F)^{-1} \int_{F} m_{j}(\xi) d H^{d}(\xi)$, where $m_{j}(\xi)$ denotes the mean value of $f_{2}$ on the intersection $L(\xi) \cap R_{j}$ (which is a line segment of length $\left.\frac{2 t_{1}}{5 C_{0}}\right)$. If $\xi \in F, u_{1} \in L(\xi) \cap R_{1}$, and $u_{2} \in L(\xi) \cap R_{2}$, then the line segment $\ell$ from $u_{1}$ to $u_{2}$ does not meet $E$ (because $\xi \notin Z^{+}$), and is of course contained in $L(\xi) \cap B(w, 5 t)$ by construction. Hence $\left|f_{2}\left(u_{1}\right)-f_{2}\left(u_{2}\right)\right| \leq \int_{\ell}\left|\nabla f_{2}\right| d H^{1} \leq \frac{8}{\sigma t_{1}^{d}}$, since $\xi \in F$. Therefore $\left|m_{1}(\xi)-m_{2}(\xi)\right| \leq \frac{8}{\sigma t_{1}^{d}}$ for all $\xi \in F$, and so

$$
\left|m_{1}-m_{2}\right| \leq \frac{8}{\sigma t_{1}^{d}}
$$


by averaging over $\xi$. This and (3.19) contradict (3.14) if $M$ is large enough, and so (3.16) holds. The analogue of (3.16) for $Z^{-}$is proved in the same manner (but using the function $f_{1}$ and the estimates (3.10) and (3.12)), and Lemma 3.15 follows.

We are now ready to define our set $E(x, t)$. For each $\xi \in Z^{+} \cap Z^{-}$, let $\eta(\xi)$ be the "first" point on $L^{+}(\xi)$ (starting from $\xi$ and going in the direction of $B_{2}$ ) that lies in $E$. By definition of $Z^{+}$this point exists and lies between $B_{1}$ and $B_{2}$. We let $E(x, t)$ be the set of points of the form $\eta(\xi), \xi \in Z^{+} \cap Z^{-}$. (See Figure 4.)

Let us check that $E(x, t)$ is a Borel set. Notice first that $Z^{+} \cap Z^{-}$is (relatively) closed in $P_{1} \cap B_{1}$. Let $\mathcal{C}$ denote the open cylinder which connects $P_{1} \cap B_{1}$ and $P_{2} \cap B_{2}$, and let $\pi$ denote the orthogonal projection onto $P_{1}$. Set $\mathcal{C}^{\prime}=\mathcal{C} \cap E \cap \pi^{-1}\left(Z^{+} \cap Z^{-}\right)$, so that $\mathcal{C}^{\prime}$ is a Borel set. Now define $\mathcal{C}_{m}^{\prime}$ to be the set of $\eta$ in $\mathcal{C}^{\prime}$ such that there is no point $e \in E$ which lies on the segment from $\pi(\eta)$ to $\eta$ and which satisfies $|e-\eta| \geq \frac{1}{m}$. Each $\mathcal{C}_{m}^{\prime}$ is a relatively open subset of $\mathcal{C}^{\prime}$, and hence is Borel. It is easy to see that $E(x, t)$ is just the intersection of the $\mathcal{C}_{m}^{\prime}$ for $m=1,2, \ldots$, and hence is Borel too.

By definition we have that the projection of $E(x, t)$ onto $P_{1}$ is $Z^{+} \cap Z^{-}$. Lemma 3.15 implies that

$$
H^{d}(E(x, t)) \geq C^{-1} t_{1}^{d}
$$

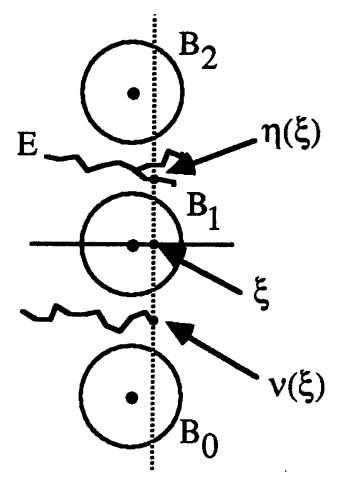

Fig. 4.

We need to be able to control the overlaps of the various $E(x, t)$ 's. Let us first establish some preliminary facts. Given $\xi \in Z^{+} \cap Z^{-}$notice that the line segment $L^{-}(\xi)$ intersects $E$ somewhere between $B_{1}$ and $B_{0}$ (by definition of $Z^{-}$). Let $\nu(\xi)$ be the first element of $E \cap L^{-}(\xi)$ on the way to $B_{0}$. Then $\nu(\xi)$ is also the first point on $E$ that one meets when one Vol. 13, $\mathrm{n}^{\circ} 4-1996$. 
starts at $\eta(\xi)$ and proceeds along $L(\xi)$ in the direction $-\theta(x, t)$. Moreover we have that

$$
\frac{2 t_{1}}{5 C_{0}} \leq \operatorname{dist}(\eta(\xi), \nu(\xi)) \leq 4 t
$$

(The first inequality follows from the fact that $2 B_{1} \subseteq D_{1}$ does not touch $E$, so that $\eta(\xi)$ and $\nu(\xi)$ are both at distance $\geq \frac{t_{1}}{5 C_{0}}$ from $\xi$.)

LEMMA 3.23. - There is a constant $C=C(\epsilon)$ such that if $(x, t),\left(x^{\prime}, t^{\prime}\right) \in$ $\mathcal{B}_{2}(\epsilon), \theta(x, t)=\theta\left(x^{\prime}, t^{\prime}\right)$, and if either $t^{\prime} \geq C(\epsilon) t$ or $\left|x-x^{\prime}\right| \geq C(\epsilon) t$, then $E(x, t) \cap E\left(x^{\prime}, t^{\prime}\right)=\emptyset$.

This is very easy. Let $\theta \in \Theta$ be given, and suppose that $\eta \in E(x, t)$ for some $(x, t) \in \mathcal{B}_{2}(\epsilon)$ such that $\theta(x, t)=\theta$. Let $\nu$ be the first element of $E$ that one hits starting from $\eta$ and going in the direction $-\theta$, so that $\nu$ corresponds to $\eta$ as above. From (3.22) we get that $\frac{2 t_{1}}{5 C_{0}} \leq \operatorname{dist}(\eta, \nu) \leq 4 t$. Thus $t$ is determined by $\eta$ to within a bounded factor, and the location of $x$ is also approximately determined, because $\eta \in B(x, 3 t)$, by construction. Lemma 3.23 follows.

Let us now finish the proof of Theorem 3.5, using Lemma 3.23 and a simple covering argument. We need to show that $\mathcal{B}_{2}(\epsilon)=\mathcal{B}(\epsilon) \backslash \mathcal{B}_{1}(\epsilon)$ is a Carleson set. Let $X$ and $R$ be given, and set $\mathcal{H}=\mathcal{B}_{2}(\epsilon) \cap$ $\left\{(E \cap B(X, R) \times(0, R]\}\right.$. Let $\Lambda$ be our usual measure $\frac{d \mu d t}{t}$ on $E \times \mathbf{R}^{+}$. We must show that

$$
\Lambda(\mathcal{H}) \leq C R^{d}
$$

for some $C$ that does not depend on $X$ or $R$.

Set $A_{j}=\left\{x \in E\right.$ : there is a $t>0$ such that $(x, t) \in \mathcal{H}$ and $2^{-j-1} R<$ $\left.t \leq 2^{-j} R\right\}$. For each $j \geq 0$ choose a maximal subset $A_{j}^{*}$ of $A_{j}$ such that $\left|x-x^{\prime}\right| \geq 2^{-j} R$ whenever $x, x^{\prime} \in A_{j}^{*}$ and $x \neq x^{\prime}$. Then $A_{j}$ is covered by the union of the balls $B\left(x, 2^{-j+1} R\right), x \in A_{j}^{*}$, and so $\mu\left(A_{j}\right) \leq C\left(2^{-j} R\right)^{d}\left(\sharp A_{j}^{*}\right)$, where $\sharp A_{j}^{*}$ denotes the number of elements in $A_{j}^{*}$. It follows that

$$
\Lambda(\mathcal{H}) \leq C \sum_{j \geq 0} \mu\left(A_{j}\right) \leq C \sum_{j \geq 0}\left(2^{-j} R\right)^{d}\left(\sharp A_{j}^{*}\right)
$$

For each $x \in A_{j}^{*}$ choose a $t \in\left(2^{-j-1} R, 2^{-j} R\right]$ so that $(x, t) \in \mathcal{H}$. Lemma 3.23 implies that no point $\eta$ in $E$ can belong to more than a bounded number $C$ of the sets $E(x, t)$ which arise in this manner (i.e., which correspond to some $j \geq 0$ and some $x \in A_{j}^{*}$ ). This uses also the fact 
that $\Theta$ is a finite set. Applying (3.21) we get that

$$
\begin{aligned}
\Lambda(\mathcal{H}) & \leq C \sum_{j \geq 0} \sum_{x \in A_{j}^{*}} H^{d}(E(x, t)) \\
& \leq C \sum_{j \geq 0} \sum_{x \in A_{j}^{*}} \mu(E(x, t)) \leq C \mu\left(\bigcup_{j, x} E(x, t)\right),
\end{aligned}
$$

using the bounded overlap property for the last inequality. It should be understood here that we are always taking $t$ to be associated to our $x$ 's as above. By our construction of $E(x, t)$ we have that $E(x, t) \subseteq B(X, 5 R)$ when $x \in A_{j}^{*}$, and so we get that $\Lambda(\mathcal{H}) \leq C \mu\left(B(X, 5 R) \leq C R^{d}\right.$, since $E$ is regular. This proves (3.24) and completes the proof of Theorem 3.5.

The fretful reader may worry about the measurability of $\mathcal{H}$ or $A_{j}$ above, but this would be pointless. One could easily work with outer measure, and in fact our argument also implies that (3.24) holds even when we replace $\mathcal{H}$ by its closure. The measurability of $\mathcal{H}$ really cannot be an issue here, because we are controlling $\Lambda(\mathcal{H})$ in terms of discrete sums that would just as easily control much fatter sets. This is similar to the situation in the proof of Sublemma 3.9.

Remark 3.27. - We know from Theorem 3.5 that the WNPC implies uniform rectifiability, but the proof that we get by invoking Theorem 2.14 is unnecessarily complicated. The main point is that we can prove directly that a set which satisfies the WNPC has "big projections", while in the proof of the "if" part of Theorem 2.14 additional constructions were required to reduce to this case. To make this precise let us begin with the following definition.

Definition 3.28. - Let $E$ be a $d$-dimensional regular set in $\mathbf{R}^{n}$. We say that $E$ has big projections if there is a constant $\theta>0$ such that for each $x \in E$ and every $r>0$ we can find a $d$-plane $P=P(x, r)$ which satisfies

$$
H^{d}\left(\Pi_{P}(E \cap B(x, r))\right) \geq \theta r^{d},
$$

where $\Pi_{P}$ denotes the orthogonal projection from $\mathbf{R}^{n}$ onto $P$.

Proposition 3.29. - If $E$ is a d-dimensional regular set in $\mathbf{R}^{d+1}$ which satisfies the WNPC, then $E$ has big projections.

Let us assume the proposition for the moment and see how it is used. Suppose that $E$ is a $d$-dimensional regular set in $\mathbf{R}^{d+1}$ which satisfies the WNPC. Theorem 3.5 implies that $E$ is locally symmetric. It is not 
very difficult to show that $E$ must then satisfy the BWGL ("bilateral weak geometric lemma"), as discussed just before Theorem 2.14. In particular $E$ must satisfy a condition called the "weak geometric lemma" (WGL) which is weaker than the BWGL by definition, and which we shall not bother to define here. (See Definition 1.16 on p. 858 of [DS2].) Since $E$ also has big projections, we can use Theorem 1.14 on p. 857 in [DS2] to conclude that $E$ is uniformly rectifiable. (We shall say more about this result soon.) This argument is very similar to a portion of the proof of the "if" part of Theorem 2.14, but it is much shorter.

Let us now prove Propostion 3.29. The basic idea occurs already in the special case of sets which satisfy Condition $B$, and the reader may find it a useful exercise to do the argument first in that simpler situation.

Suppose that $E$ satisfies the WNPC, and let $(x, r) \in E \times \mathbf{R}^{+}$be given. Let $C_{0}$ be as in the definition of the WNPC, and let $M$ be a large constant, to be chosen soon. We claim first that there is a constant $C_{1}$, which does not depend on $(x, r)$, and a pair $(y, t) \in E \times \mathbf{R}^{+}$such that $B(y, t) \subseteq B(x, r), t \geq \frac{r}{C_{1}}$, and

$$
(y, t) \notin \mathcal{B}\left(C_{0}, 1, M\right)
$$

This follows from the fact that $\mathcal{B}\left(C_{0}, 1, M\right)$ is a Carleson set. If there were no such $(y, t)$, then $\mathcal{B}\left(C_{0}, 1, M\right)$ would contain the whole set $(E \cap$ $\left.B\left(x, \frac{r}{2}\right)\right) \times\left[\frac{r}{C_{1}}, \frac{r}{2}\right]$. Since the $\Lambda$ measure of this set is $\mu\left(E \cap B\left(x, \frac{r}{2}\right)\right) \log \frac{C_{1}}{2}$ (where $\Lambda$ is our usual product measure on $E \times \mathbf{R}^{+}$), we would get a contradiction if $C_{1}$ is large enough.

From (3.30) and the definition of $\mathcal{B}\left(C_{0}, 1, M\right)$ we obtain that there are two reasonably large balls $B_{1}$ and $B_{2}$ contained in $B(y, t)$ and a function $f \in C^{\infty}(B(y, t) \backslash E)$ for which (3.3) fails. Let $P=P(x, r)$ be a $d$-plane which is orthogonal to the line which joins the centers of $B_{1}$ and $B_{2}$. We claim that

$$
H^{d}(\Pi(E \cap B(y, t))) \geq C^{-1} t^{d}
$$

if $M$ is chosen correctly, where $\Pi=\Pi_{P}$ is as in Definition 3.28 and $C$ is a positive constant that does not depend on $(x, r)$. The point is that if $\Pi(E \cap B(y, t))$ did not contain most of $\Pi\left(\frac{1}{2} B_{1}\right)$, say, then there would be many parallel line segments that join $\frac{1}{2} B_{1}$ to $\frac{1}{2} B_{2}$ without touching $E$. (See Figure 5.) This would lead to a contradiction, because we could argue as in the proof of Lemma 3.15 to get a bound on the difference between the averages of $f$ over $B_{1}$ and $B_{2}$ and thereby conclude that (3.3) does hold 




Fig. 5 .

(if $M$ is large enough). We omit the details. (This result is not essential to the purposes of this paper anyway.)

Of course Propostion 3.29 follows from (3.31), because of our choice of $(y, t)$.

Let us say a little more about the criterion for uniform rectifiability given in [DS2]. To do this we need another definition.

Definition 3.32. - A $d$-dimensional regular set $E \subseteq \mathbf{R}^{n}$ has big pieces of Lipschitz graphs (BPLG) if there exist $M \geq 0$ and $\theta>0$ so that for each $x \in E$ and $r>0$ we can find a subset $\Gamma$ of $\mathbf{R}^{n}$ such that

$$
H^{d}(E \cap B(x, r) \cap \Gamma) \geq \theta r^{d}
$$

and

$\Gamma$ is a rotation of the graph of a function

$$
A: \mathbf{R}^{d} \rightarrow \mathbf{R}^{n-d} \text { with Lipschitz norm } \leq M \text {. }
$$

We shall sometimes refer to a set $\Gamma$ as in (3.34) as a Lipschitz graph, or as an $M$-Lipschitz graph when we want to be precise about the constants.

The precise statement of Theorem 1.14 in [DS2] is that if a regular set has big projections and satisfies the weak geometric lemma then it has BPLG. It is not so obvious that BPLG implies the definition of uniform rectifiability given in Section 2, but it follows from the main result in [DS1]. A more direct proof can be obtained by using Theorem 2.29 on p. 336 of [DS3] to pass from BPLG to the existence of a "corona decomposition" (which we shall not define here) and then using a smaller piece of [DS1] to get uniform rectifiability. (This smaller piece is contained entirely in Section 18 of [DS1].) When $d=1$ there are reasonably direct geometric methods for producing a regular curve $\Gamma \supseteq E$ when $E$ has BPLG. The first person to draw our attention to this fact was Peter Jones, but we do not know of anyplace where this has been recorded. Let us briefly sketch the argument.

Proposition 3.35. - A one-dimensional regular set in $\mathbf{R}^{n}$ which has $B P L G$ is contained in a regular curve. 
We could also deal with weaker conditions than BPLG (such as BPLI, see [DS3]), but for simplicity of exposition we restrict ourselves to this case.

Suppose that $E \subseteq \mathbf{R}^{n}$ is one-dimensional and has BPLG. Let us first show that for each $X \in E$ and $R>0$ there is a connected compact set $\gamma$ in $\mathbf{R}^{n}$ which contains $E \cap B(X, R)$ and satisfies $H^{1}(\gamma) \leq C R$.

We shall produce this set $\gamma$ as the limit of a sequence $\left\{\gamma_{j}\right\}$ of compact connected sets with finite length. Set $E_{0}=E \cap B(X, R)$. We begin by taking $\gamma_{0}$ to be a curve in $\bar{B}(X, R)$ such that $H^{1}\left(\gamma_{0} \cap E_{0}\right) \geq \theta R$ and $H^{1}\left(\gamma_{0}\right) \leq C R$, where $\theta$ is as in the definition of BPLG. We can do this because $E$ has BPLG. More precisely, because of the BPLG condition there is a curve like $\gamma_{0}$ which is an arc of an $M$-Lipschitz graph but which is not necessarily contained in $\bar{B}(X, R)$, but we can modify it slightly to get a curve with all the required properties.

Now suppose that we have already constructed $\gamma_{0}, \ldots, \gamma_{j}$, and let us construct $\gamma_{j+1}$. Set $U_{j}=E_{0} \backslash \gamma_{j}$ and, for each $x \in U_{j}$, let $B(x)$ denote the closed ball centered at $x$ and wih radius $\delta(x)=\operatorname{dist}\left(x, \gamma_{j}\right)$. Notice that $\delta(x)<2 R$ when $x \in U_{j}$. By the standard Vitali covering argument (as on p. 9, 10 of [St]) we can find a subset $A$ of $U_{j}$ such that the balls $B(x), x \in A$, are pairwise disjoint and the balls $5 B(x), x \in A$, cover $U_{j}$. Fix $x \in A$, and let us show that there is a compact connected set $\alpha_{x} \subseteq B(x)$ such that $\alpha_{x}$ touches $\gamma_{j}$,

$$
H^{1}\left(\alpha_{x} \cap E \cap \frac{1}{2} B(x)\right) \geq \theta \delta(x),
$$

and

$$
H^{1}\left(\alpha_{x}\right) \leq C \delta(x)
$$

Since $E$ has BPLG we can find an $M$-Lipschitz graph $\xi_{x}$ which satisfies the analogue of (3.36). Let $\xi_{x}{ }^{\prime}$ be the smallest arc of $\xi_{x}$ which contains $\xi_{x} \cap B(x)$. We can modify $\xi_{x}{ }^{\prime}$ slightly to get a curve in $B(x)$ which contains $\xi_{x} \cap B(x)$ and which has roughly the same length as $\xi_{x}{ }^{\prime}$. We then add to this modified curve a line segment which connects it to $\gamma_{j}$ to get $\alpha_{x}$. Note that we can choose this line segment to lie in $B(x)$, since $B(x)$ touches $\gamma_{j}$ by definition.

Let $\gamma_{j+1}$ to be the union of $\gamma_{j}$ and the sets $\alpha_{x}, x \in A$. It is easy to see that $\gamma_{j+1}$ is closed, connected, and has finite length. We also have

$$
\gamma_{j+1} \backslash \gamma_{j} \subseteq B(X, 3 R)
$$


(since $\alpha_{x} \subseteq B(x) \subseteq B(X, 3 R)$ for all $x \in A$ ) and

$$
\begin{aligned}
H^{1}\left(\gamma_{j+1} \backslash \gamma_{j}\right) & \leq \sum_{x \in A} H^{1}\left(\gamma_{x}\right) \leq C \sum_{x \in A} \delta(x) \\
& \leq C \sum_{x \in A} H^{1}\left(\alpha_{x} \cap E \cap \frac{1}{2} B(x)\right) \\
& \leq C H^{1}\left(\left(\gamma_{j+1} \backslash \gamma_{j}\right) \cap E\right)
\end{aligned}
$$

because the balls $\frac{1}{2} B(x), x \in A$, are pairwise disjoint and do not touch $\gamma_{j}$. On the other hand, from (3.36) we get

$$
H^{1}\left(\left(\gamma_{j+1} \backslash \gamma_{j}\right) \cap E\right) \geq \theta \sum_{x \in A} \delta(x) \geq C^{-1} \theta H^{1}\left(U_{j}\right)
$$

using also the fact that $U_{j}$ is covered by the balls $5 B(x), x \in A$, and the assumption that $E$ is regular.

By repeating this construction indefinitely we obtain our sequence $\left\{\gamma_{j}\right\}$. Note that it could happen that $U_{j}=\emptyset$ for some $j$, in which case $\gamma_{j+1}=\gamma_{j}$. From (3.38), (3.39), and the corresponding properties for $\gamma_{0}$ we conclude that $\gamma_{j} \subseteq B(X, 3 R)$ and $H^{1}\left(\gamma_{j}\right) \leq C R$ for all $j$. Let $\gamma$ be the closure of the union of the $\gamma_{j}$ 's. It is easy to see that $\gamma \subseteq \bar{B}(X, 3 R)$ and that $\gamma$ is compact and connected. Let us check that $\gamma \supseteq E_{0}$. Suppose not, so that there is a point $y \in E_{0}$ that does not lie in $\gamma$. Then there is a $t>0$ such that $B(y, t) \cap E \subseteq U_{j}$ for all $j$. However, (3.40) and (3.39) imply that $H^{1}\left(U_{j}\right) \rightarrow 0$ as $j \rightarrow \infty$, which contradicts the preceding statement. Thus $\gamma \supseteq E_{0}$.

We also have that $H^{1}(\gamma) \leq C R$, i.e., the limits points that we are adding do not have too much mass. This is not hard to prove, using the definition of Hausdorff measure and the fact that the $\gamma_{j}$ 's are connected. We omit the details. Thus we have achieved our first goal, of showing that $E \cap B(X, R)$ is contained in a connected compact set $\gamma$ with $H^{1}(\gamma) \leq C R$. Of course we can always modify $\gamma$ slightly to get $\gamma \subseteq \bar{B}(X, R)$ also.

Next let us show that for each $X \in E$ and $R>0$ there is a compact connected set $\Gamma$ such that $B(X, R) \cap E \subseteq \Gamma \subseteq \bar{B}(X, R)$ and

$$
C_{1}^{-1} r \leq H^{1}(\Gamma \cap B(x, r)) \leq C_{1} r
$$

for all $x \in \Gamma$ and $r \in(0, R]$.

One way to approach this task is to build $\Gamma$ by hand, in the same manner as above, but taking more care to ensure that the curves $\alpha_{x}$ do not pile 
up too much. There is an elegant and amusing alternative to this method which was pointed out to us by J. M. Morel and S. Solimini and which we describe instead. (See $[\mathrm{MoS}]$ for details, and note that they also give a similarly elegant but slightly indirect argument for the existence of the curves $\gamma$ constructed above.)

Let $X \in E$ and $R>0$ be given. Let $\Gamma$ be a compact connected set which satisfies $B(X, R) \cap E \subseteq \Gamma \subseteq \bar{B}(X, R)$ with $H^{1}(\Gamma)$ as small as possible. Such a minimizer exists, because there are suitable lower semicontinuity theorems for the $H^{1}$-measure of compact connected sets (see [MoS]). Note that our previous construction implies that there exist competitors for this minimization which have finite length. Let us check that such a minimizing $\Gamma$ must satisfy (3.41).

Let $x \in \Gamma$ and $r \in(0, R]$ be given. The lower bound in (3.41) follows from connectedness, and so we only need to verify the upper bound. Suppose, to the contrary, that $H^{1}(\Gamma \cap B(x, r)) \geq C_{1} r$ and that $C_{1}$ is very large. By our earlier construction we can find a compact connected set $\gamma$ such that $\gamma \supseteq E \cap B(x, r) \cap B(X, R)$ and $H^{1}(\gamma) \leq C r$. We can modify $\gamma$ slightly to get also that $\gamma \subseteq B(x, r) \cap B(X, 2 R)$ (and without increasing the length of $\gamma$ too much). If $\Gamma \subseteq B(x, r)$, then $\gamma$ satisfies the same requirements as $\Gamma$ but has less $H^{1}$ measure (if $C_{1}$ is large enough), a contradiction. If $\Gamma \nsubseteq B(x, r)$, then let $\Gamma^{\prime}$ be the union of $\Gamma \backslash B(x, r), \gamma$, and a line segment which connects $\gamma$ to a point in $\Gamma \cap \partial B(x, r)$. In this case $\Gamma^{\prime}$ satisfies the same requirements as $\Gamma$ but has smaller $H^{1}$ measure (if $C_{1}$ is large enough). Thus we conclude that $\Gamma$ satisfies (3.41).

There is a more constructive version of this method for producing $\Gamma$ that is worth mentioning. We start with any compact connected set $\Gamma$ with finite length and which satisfies $B(X, R) \cap E \subseteq \Gamma \subseteq \bar{B}(X, R)$, and we make a sequence of modifications to it to get a set which also satisfies (3.41). Since $\Gamma$ is connected the lower bound in (3.41) is automatic, and so we need only concern ourselves with the upper bound. Suppose that $x \in \Gamma$ and $r \in(0, r]$ are bad for (3.41), so that $H^{1}(\Gamma \cap B(x, r))>C_{1} r$, and assume that $x, r$ are chosen so that $r$ is also approximately as large as possible. If $C_{1}$ is large enough, then we can modify $\Gamma$ to get a compact connected set $\Gamma^{\prime}$ in the same manner as above, in such a way that $B(X, R) \cap E \subseteq \Gamma^{\prime} \subseteq \bar{B}(X, R)$ and $H^{1}\left(\Gamma^{\prime}\right) \leq H^{1}(\Gamma)-r$. We then apply the same procedure to $\Gamma^{\prime}$, and then we keep repeating it as long as (3.41) is not satisfied (with a reasonably large but fixed choice of $C_{1}$ ). If this process does not stop in a finite number of steps, it will still "converge", because in each step we are winning so much length that the size of the modifications must go to 0 . In either way 
we end up with a set $\Gamma$ which satisfies (3.41) and which has the other required properties.

We are nearly finished with our sketch of the proof of Proposition 3.35. The "local" fact that we just verified has a simple global version, to wit, $E$ is contained in a connected 1-dimensional regular set in $\mathbf{R}^{n}$. This is not very hard to show, by applying the preceding fact to larger and larger balls and connecting the $\Gamma$ 's that result in a suitable manner. One needs to be a little careful, to avoid having too much mass accumulate anywhere, but this is not difficult to arrange.

It remains to show that a connected 1-dimensional regular set is contained in a regular curve. For compact sets there are stronger results: compact connected sets with finite $H^{1}$ measure are basically the same thing as curves, in the sense that they can be realized as the image of an interval under a Lipschitz mapping, and this mapping can even be chosen so that the parameterized length is not too much larger than the $H^{1}$ measure of the original set. See Theorem 1.8 on p. 6 of [DS3] for a proof of this classical fact. The same method can be used to show that if a compact set satisfies estimates like (3.41), then the parameterization can be chosen to satisfy (2.3) and (2.4). Thus we conclude that if $E$ is a connected 1-dimensional regular set, then any compact connected subset of it can be realized as a subset of a regular curve. It is not very hard to use this to show that $E$ itself is contained in a regular curve. (Notice that connected 1-dimensional sets cannot always be realized as a regular curve, rather than merely as a subset. The union of two perpendicular lines in the plane won't work, for instance.)

This completes the discussion of the proof of Proposition 3.35.

Although the BPLG implies uniform rectifiability, the converse is not true. There are one-dimensional sets in the plane which are uniformly rectifiable but which do not satisfy BPLG. (See [Hr].) Let us therefore record the following.

THEOREM 3.42. - A d-dimensional regular set in $\mathbf{R}^{d+1}$ which satisfies the WNPC has BPLG.

This follows from the proof of the fact that the WNPC implies uniform rectifiability which is outlined just after Proposition 3.29. That argument uses Theorem 1.14 in [DS2], which actually gives BPLG and not just uniform rectifiability.

Note that a regular set satisfies BPLG if it is uniformly rectifiable and has big projections. This can be derived from the main result of [Js], or Theorem 1.14 in [DS2]. The converse is also true. We have already seen that BPLG implies uniform rectifiability, and the fact that BPLG implies big projections follows from the definitions.

Vol. 13, $\mathrm{n}^{\circ} 4-1996$. 
Note that the BPLG does not imply the WNPC. The same example as mentioned shortly after Theorem 3.5 applies here too (i.e., a $d$-plane with a lattice of holes of fixed size).

Remark 3.43. - The proof of the fact that the local symmetry condition implies uniform rectifiability actually implies a stronger result. Let $E$ be a $d$-dimensional regular set in $\mathbf{R}^{n}$. Then there is a (small) $\epsilon_{0}>0$, which depends only on the dimensions and the regularity constant for $E$, such that $E$ is uniformly rectifiable if $\mathcal{B}\left(\epsilon_{0}\right)$ is a Carleson set. (Compare with Definition 2.12.) This is a small variant of Remark 2.5 on p. 98 of [DS3]. There is a similar strengthening of Theorem 1.14 in [DS2]; the result still holds if, instead of requiring the "weak geometric lemma" at full strength, one weakens it in the same manner. Similarly, in deriving uniform rectifiability from the WNPC, it is actually enough to assume that there is a $C_{0} \geq 1$ such that $\mathcal{B}\left(C_{0}, k, M\right)$ is a Carleson set for a single sufficiently large choice of $k, M$ which depends on $C_{0}, d$, and the regularity constant for $E$. As a practical matter, the selection of $k$ and $M$ depends on the analogous choice of $\epsilon_{0}$ mentioned above, or its counterpart for Theorem 1.14 in [DS2] (which is essentially the same issue).

\section{SINGULARITY SETS OF SOME BV FUNCTIONS}

Let $E$ continue to be a $d$-dimensional regular set in $\mathbf{R}^{d+1}$. We need a definition before we state the main result of this section.

Definition 4.1. - A "Carleson measure with respect to $E$ " is a nonegative Borel measure $\nu$ on $\mathbf{R}^{d+1}$ such that

$$
\nu(B(x, r)) \leq C_{0} r^{d} \quad \text { for all } \quad x \in E \text { and } r>0 .
$$

The smallest constant $C_{0}$ for which (4.2) holds is called the Carleson norm of $\nu$ and will be denoted by $\|\nu\|_{C}$.

A simple example is the restriction of Hausdorff measure $H^{d}$ to $E$. In fact, the restriction of $H^{d}$ to any $d$-dimensional regular set defines a Carleson measure with respect to any other $d$-dimensional regular set. Carleson measures can also be more smeared-out. For instance, given a $t>0$, take $\nu$ to be Lebesgue measure on $\left\{x \in \mathbf{R}^{d+1}: \operatorname{dist}(x, E)<t\right\}$ divided by $t$. The regularity of $E$ implies that this is a Carleson measure with respect to $E$, with norm bounded independently of $t$. 
THEOREM 4.3. - Let $E$ be a d-dimensional regular set in $\mathbf{R}^{d+1}$ and let $f$ be a locally integrable function on $\mathbf{R}^{d+1}$. Suppose that $\nabla f$ (the distributional gradient of $f$ ) is of the form $h d \nu$, where $h$ is a bounded vector-valued (Borel measurable) function and $\nu$ is a Carleson measure with respect to $E$. Suppose also that there is a constant a $>0$ so that

$$
\inf _{\alpha \in \mathbf{R}} r^{-d-1} \int_{B(x, r)}|f(y)-\alpha| d y \geq a \quad \text { for all } x \in E \text { and } r>0 .
$$

Then $E$ is uniformly rectifiable.

Condition (4.4) says that $f$ is discontinuous at points in $E$, with a uniform lower bound on the size of the jump. Theorem 4.3 should be compared with the classical fact (see conclusion (16) of Theorem 4.5.9 on p. 483 of [Fe] and Chapter 4 of [Gi]) that if $f$ is of bounded variation - so that $\nabla f$ is a (vector-valued) measure of finite mass - then the "singular set" of non-Lebesgue points of $f$ is rectifiable. In other words, Theorem 4.3 is a uniformized version of this classical result.

Theorem 4.3 is a close relative of the fact that Condition B (see Definition 2.15) implies uniform rectifiability. If $E$ satisfies Condition B and if we assume for simplicity that $E$ has exactly two complementary components, then the characteristic function of either complementary component satisfies the conditions required of $f$ in Theorem 4.3. Indeed, the Carleson condition on $|\nabla f|$ can be derived easily from the assumption that $E$ is regular (because, as a measure, $|\nabla f|$ is dominated by the restriction of $H^{d}$ to $E$ ), and (4.4) follows from the assumption that $E$ satisfies Condition B. In this special situation (where $f$ is locally constant on $\mathbf{R}^{d+1} \backslash E$ ) Condition B is implied by (4.4) too, but in general the hypotheses of Theorem 4.3 do not imply Condition B, because they allow $E$ to be a half-plane, or a hyperplane with a hole in it, for instance. (Exercise.)

Theorem 4.3 has a converse: if $E$ is a regular set of codimension 1 which is uniformly rectifiable, then there is a function $f$ which satisfies the conditions above. The precise statement is given in Theorem 4.51 below. This converse provides an appealing substitute for the false converse to the theorem that Condition B implies uniform rectifiability.

We shall derive Theorem 4.3 from a generalization (Theorem 4.13) of the fact that Condition $\mathrm{B}$ implies uniform rectifiability. This generalization will be based on a variant of Condition B (called Condition C) which allows a limited amount of holes, ends, and other bad behavior forbidden by Condition B. To state this generalization we need some auxiliary definitions.

Let $E$ be a $d$-dimensional regular set in $\mathbf{R}^{d+1}$, and let $b(x)$ be a nonnegative continuous function on $\Omega=\mathbf{R}^{d+1} \backslash E$. If $\gamma$ is a rectifiable 
curve in $\mathbf{R}^{d+1}$, define its $b$-length by

$$
\begin{aligned}
L_{b}(\gamma) & =\int_{\gamma} b d H^{1} & & \text { if } \gamma \subseteq \Omega \\
& =+\infty & & \text { if } \gamma \text { intersects } E .
\end{aligned}
$$

Given $x, y \in \Omega$, set

(4.6) $D_{b}(x, y)=\inf \left\{L_{b}(\gamma): \gamma\right.$ is a rectifiable curve that connects $x$ to $\left.y\right\}$.

Notice that

$$
D_{b}(x, z) \leq D_{b}(x, y)+D_{b}(y, z) \quad \text { for all } \quad x, y, z \in \Omega \text {. }
$$

The quantity $D_{b}(x, y)$ measures the separation between $x$ and $y$ caused either by $E$ or $b$. If $x$ and $y$ lie in different components of $\Omega$, then $D_{b}(x, y)=\infty$. If $x, y$ lie in the same component of $\Omega$, then $D_{b}(x, y)$ vanishes when $b \equiv 0$, and otherwise it measures the separation caused by $b$. Thus the function $b$ allows us to have a more flexible notion of separation of points in $\Omega$, and to create more "components" of $\Omega$. However, we want to limit the amount of separation that $b$ can contribute, and so we shall impose the following constraints on $b$ :

$$
0 \leq b(x) \leq C_{1} \operatorname{dist}(x, E)^{-1} \quad \text { for some } C_{1}>0 \text { and all } x \in \Omega
$$

$$
d \nu(x)=b(x) d x \quad \text { is a Carleson measure with respect to } E \text {. }
$$

Condition (4.8) simply prevents $b$ from being too wild locally in $\Omega$. Condition (4.9) is much more interesting. It basically says that $b(x) d x$ is no larger in terms of its overall mass distribution than $H^{d}$ on a $d$-dimensional regular set, although it will be smeared out locally in $\Omega$.

Definition 4.10. - A $d$-dimensional regular set $E \subseteq \mathbf{R}^{d+1}$ satisfies "Condition C" if there is a continuous function $b(x)$ on $\Omega=\mathbf{R}^{d+1} \backslash E$ which satisfies (4.8) and (4.9), and a constant $\eta>0$ such that for every $x \in E$ and $t>0$ we can find points $y_{1}, y_{2} \in B(x, t) \backslash E$ which satisfy

$$
\operatorname{dist}\left(y_{i}, E\right) \geq \eta t \quad \text { for } \quad i=1,2 \text {, }
$$

and

$$
D_{b}\left(y_{1}, y_{2}\right) \geq \eta
$$


Notice that Condition B (Definition 2.15) implies Condition C with $b \equiv 0$. The main difference between Condition $\mathrm{B}$ and Condition $\mathrm{C}$ is that we replace the requirement that $y_{1}$ and $y_{2}$ lie in different components of $\Omega$ with the weaker assumption (4.12). In other words, we allow points to be separated by $b$ even if they are not separated by $E$, although (4.9) implies that this cannot happen too often.

For simple examples, take $E$ to be a hyperplane with a ball removed, or a $d$-dimensional half-plane. Then $E$ satisfies Condition $\mathrm{C}$ but not Condition B. (It is a good exercise to check this. In these examples one takes $b(x)$ to be $\operatorname{dist}(x, E)^{-1}$ on certain parts of the complement of $E$ near the trouble spots, and 0 otherwise.)

THEOREM 4.13. - If a d-dimensional regular set $E \subseteq \mathbf{R}^{d+1}$ satisfies Condition $C$, then it is locally symmetric (and hence uniformly rectifiable, by Theorem 2.14).

We shall eventually derive Theorem 4.3 from Theorem 4.13 by showing that the hypotheses of Theorem 4.3 imply that $E$ satisfies Condition C. Basically we shall take $b(x)$ to be $|\nabla f|$, except for an initial smoothing of $f$. The uniform discontinuity condition (4.4) will give us the points $y_{1}$ and $y_{2}$ as in Definition 4.10.

Let us now prove Theorem 4.13. Let $E$ be a regular set which satisfies Condition $\mathrm{C}$, and set $\Omega=\mathbf{R}^{d+1} \backslash E$. Without loss of generality we may assume that the constant $C_{1}$ in (4.8) and the Carleson norm of $d \nu(x)=b(x) d x$ are both $\leq 1$. (Otherwise divide $b$ by a suitable constant, and then divide $\eta$ by the same constant.) Let $\eta$ be as in (4.11) and (4.12), and notice that $\eta$ must be $<1$. Let $\epsilon>0$ be given, and let $\mathcal{B}(\epsilon)$ be the bad set for the local symmetry condition defined by (2.13).

The proof of Theorem 4.13 will be along the same lines as for the analogous results for Condition B or the WNPC. We shall associate to each $(x, t) \in \mathcal{B}(\epsilon)$ a set $E(x, t) \subseteq E$ and a set $F(x, t) \subseteq \Omega$ in such a way that we control their overlaps for different $(x, t)^{\prime}$ 's. We shall control the total mass of the $E(x, t)$ 's and $F(x, t)$ 's in terms of the regularity condition (2.1) and the Carleson condition (4.9). We shall also show that for each $(x, t) \in \mathcal{B}(\epsilon)$ one of $E(x, t)$ and $F(x, t)$ is not too small. The combination of these estimates will tell us that there are not too many $(x, t)$ 's, i.e., that $\mathcal{B}(\epsilon)$ is a Carleson set.

Let $(x, t) \in \mathcal{B}(\epsilon)$ be given. By definition of $\mathcal{B}(\epsilon)$ there are points $w, z \in E \cap B(x, t)$ such that $\operatorname{dist}(2 z-w, E)>\epsilon t$. Set $t_{1}=\frac{\eta \epsilon t}{100}$ and apply Condition $\mathrm{C}$ to the pair $\left(z, t_{1}\right)$. We conclude that there is a point $y_{1} \in B\left(z, t_{1}\right)$ such that

$$
\operatorname{dist}\left(y_{1}, E\right) \geq \eta t_{1}
$$

Vol. $13, \mathrm{n}^{\circ} 4-1996$. 
and

$$
D_{b}\left(y_{1}, 2 z-w\right) \geq \frac{\eta}{2}
$$

(Actually, we get two points which satisfy (4.12), but we use the one for which $D_{b}(y, 2 z-w)$ is larger. Remember (4.7).) Applying the same argument to the pair $\left(w, t_{1}\right)$ we get a point $y_{2} \in B\left(w, t_{1}\right)$ such that

$$
\operatorname{dist}\left(y_{2}, E\right) \geq \eta t_{1}
$$

and

$$
D_{b}\left(y_{2}, y_{1}\right) \geq \frac{\eta}{2}
$$

Notice that (4.17) and (4.8) (with $C_{1}=1$ ) imply that $\left|y_{2}-y_{1}\right| \geq$ $\frac{\eta}{4} \operatorname{dist}\left(y_{i}, E\right)$ for $i=1,2$.

Next we want to choose a pair of balls $B_{1}=B\left(z_{1}, \frac{\eta^{2} t_{1}}{100}\right)$ and $B_{2}=B\left(z_{2}, \frac{\eta^{2} t_{1}}{100}\right)$ such that $2 B_{j} \subseteq B\left(y_{j}, \frac{\eta^{2} t_{1}}{40}\right)$ for $j=1,2$. We have a little room to move $B_{1}$ and $B_{2}$, and so we can choose them in such a way that the unit vector $\theta(x, t)=\frac{z_{2}-z_{1}}{\left|z_{2}-z_{1}\right|}$ lies in a fixed finite set $\Theta=\Theta(\epsilon, \eta)$ of directions (which does not depend on $x, t, z, w$, etc.).

Set $z_{0}=2 z_{1}-z_{2}$ and $B_{0}=B\left(z_{0}, \frac{\eta^{2} t_{1}}{100}\right)$. Because of our choices of $y_{1}$ and $y_{2}$ we get that

$$
B_{0} \subseteq B\left(2 z-w, 4 t_{1}\right)=B\left(2 z-w, \frac{4 \eta \epsilon t}{100}\right) .
$$

Thus $\operatorname{dist}(u, E) \geq \frac{\epsilon t}{2}$ when $u \in B_{0}$, because of the way $w$ and $z$ were chosen. Using (4.8) with $C_{1}=1$ we obtain

$$
D_{b}(u, 2 z-w) \leq \frac{\eta}{10} \quad \text { when } u \in B_{0}
$$

Similarly, using (4.14) and (4.16) we get

$$
\begin{array}{ll}
D_{b}\left(u, y_{1}\right)<\frac{\eta}{10} & \text { when } u \in 2 B_{1} \\
D_{b}\left(u, y_{2}\right)<\frac{\eta}{10} & \text { when } u \in 2 B_{2} .
\end{array}
$$

These estimates, together with (4.15), (4.17), (4.7), and the definition of $D_{b}$ imply that

$$
L_{b}(\gamma)>\frac{\eta}{4} \quad \text { whenever } \gamma \text { connects } B_{0} \text { to } B_{1} \text { or } B_{2} \text { to } B_{1}
$$


We are now ready to define our sets $E(x, t) \subseteq E$ and $F(x, t) \subseteq \Omega$. For $j=0,1,2$, let $P_{j}$ be the hyperplane through $z_{j}$ which is orthogonal to $\theta(x, t)$. For $\xi \in P_{1} \cap B_{1}$, let $L_{+}(\xi)$ be the half-line emanating from $\xi$ in the direction $\theta(x, t)$. (See Figure 3 in Section 3.) Also denote by $\mu(\xi)$ the point $\mu \in L_{+}(\xi)$ closest to $\xi$ such that the line segment $\gamma=[\xi, \mu]$ connecting $\xi$ to $\mu$ satisfies $L_{b}(\gamma) \geq \frac{\eta}{5}$. This point $\mu(\xi)$ exists and lies between $P_{1}$ and $P_{2}$ because of (4.22). The segment $[\xi, \mu(\xi))$ does not intersect $E$, since otherwise $\mu(\xi)$ would not be as close to $\xi$ as possible. (Don't forget the second possibility in (4.5).) Set

$$
E(x, t)=\left\{\mu \in E: \text { there is a } \xi \in P_{1} \cap B_{1} \text { such that } \mu=\mu(\xi)\right\}
$$

and

$$
F(x, t)=\bigcup_{\xi \in P_{1} \cap B_{1}} \gamma(\xi)
$$

where

$$
\gamma(\xi)=(\xi, \mu(\xi))
$$

LEMMA 4.26. $-F(x, t)$ is an open subset of $\Omega$, and $E(x, t)$ is a Borel set.

Let $\Pi$ be the orthogonal projection onto $P_{1}$, and let $T$ denote the part of $\Pi^{-1}\left(P_{1} \cap B_{1}\right)$ that lies (strictly) between $P_{1}$ and $P_{2}$. The first part of the lemma is easy, because $F(x, t)$ is the set of points $\mu \in T$ for which the segment $[\Pi(\mu), \mu]$ does not intersect $E$ and such that the integral of $b$ on this segment is $<\frac{\eta}{5}$. For the second part of the lemma we observe that $E(x, t)$ is the set of points $\mu \in E \cap T$ such that $\mu-\frac{1}{n} \theta(x, t)$ lies in $F(x, t)$ for all sufficiently large $n$. This implies easily that $E(x, t)$ is a Borel set.

Notice that $\mu(\xi)$ is a Borel measurable function. To see this it suffices to check that $(\mu(\xi)-\xi) \cdot \theta(x, t)$ is Borel measurable. This last is actually an upper semicontinuous function, since $F(x, t)$ is open.

$$
\text { LeMma 4.27. }-H^{d}(E(x, t))+\nu(F(x, t)) \geq C(\epsilon, \eta)^{-1} t^{d} \text {. }
$$

Set $A_{1}=\left\{\xi \in P_{1} \cap B_{1}: \mu(\xi) \in E\right\}$ and $A_{2}=P_{1} \cap B_{1} \backslash A_{1}$, so that both sets are measurable, since $\mu(\xi)$ is a measurable function. Observe that $A_{1}=\Pi(E(x, t))$, and hence $H^{d}(E(x, t)) \geq H^{d}\left(A_{1}\right)$. On the other hand, for each $\xi \in A_{2}$ we have that the integral of $b$ over $[\xi, \mu(\xi))$ is equal to $\frac{\eta}{5}$. Integrating this over $A_{2}$ gives $\nu(F(x, t)) \geq \frac{\eta}{5} H^{d}\left(A_{2}\right)$. (Remember that $d \nu=b(x) d x$.) Lemma 4.27 follows from these two estimates and the definition of $t_{1}$. 
Our next goal is to control the overlaps of the $E(x, t)$ 's and the $F(x, t)$ 's. To do this we shall need the following lemma, which tells about what happens when we look "down" (in the direction of $-\theta(x, t)$ ) instead of "up".

Lemma 4.28. - Given $p \in E(x, t) \cup F(x, t)$, let $\alpha(p)$ be the smallest nonnegative real number $\alpha$ such that $L_{b}((p, p-\alpha \theta(x, t)]) \geq \frac{\eta}{4}$. Then

$$
\frac{\eta^{2} t_{1}}{100} \leq \alpha(p) \leq 5 t
$$

Let $p \in E(x, t) \cup F(x, t)$ be given, and observe that $L_{b}((p, \Pi(p)]) \leq \frac{\eta}{5}$, by definition of $E(x, t), F(x, t)$, and $\mu(\xi)$. This implies that $\alpha(p)>0$. On the other hand, if $u$ is the point on $P_{0}$ of the form $u=p-\beta \theta(x, t)$ for some $\beta>0$, then $L_{b}((\Pi(p), u))>\frac{\eta}{4}$, because of (4.22). The conclusion of all this is that $\alpha(p)$ exists, is positive and finite, and that $p-\alpha(p) \theta(x, t)$ lies somewhere between $P_{1}$ and $P_{0}$. The second inequality in (4.29) follows from this and the fact that $p$ lies between $P_{2}$ and $P_{1}$. To get the first inequality it is enough to notice that at least one of the two points $p$ and $p-\alpha(p) \theta(x, t)$ must lie outside $2 B_{1}$, because of (4.20). (See Figure 6.)



Fig. 6.

LEMmA 4.30. - There is a constant $C(\epsilon, \eta)$ such that if $(x, t),\left(x^{\prime}, t^{\prime}\right) \in$ $\mathcal{B}(\epsilon)$ satisfy $\theta(x, t)=\theta\left(x^{\prime}, t^{\prime}\right)$ and either $t^{\prime} \geq C(\epsilon, \eta)$ tor $\left|x^{\prime}-x\right| \geq C(\epsilon, \eta) t$, then $E(x, t) \cap E\left(x^{\prime}, t^{\prime}\right)=\emptyset$ and $F(x, t) \cap F\left(x^{\prime}, t^{\prime}\right)=\emptyset$.

This is an easy consequence of Lemma 4.28. Notice first that $E(x, t) \cup F(x, t) \subset B(x, 4 t)$. Thus if $\left|x-x^{\prime}\right| \geq 10 \max \left(t, t^{\prime}\right)$, then $B(x, 4 t)$ and $B\left(x^{\prime}, 4 t^{\prime}\right)$ are disjoint, and we are in business. Suppose now that $t$ is much larger than $t^{\prime}$ (or vice-versa), and that there is a point $p$ in one of the forbidden intersections. If one defines $\alpha(p)$ as in Lemma 4.28 for both $(x, t)$ 
and $\left(x^{\prime}, t^{\prime}\right)$, then one should get the same answer, since $\theta(x, t)=\theta\left(x^{\prime}, t^{\prime}\right)$, but this contradicts (4.29) if $t$ is sufficiently large compared to $t^{\prime}$. Lemma 4.30 follows.

To conclude the proof of Theorem 4.13 we use the same argument as employed in Section 3 to derive Theorem 3.5 from Lemma 3.23. Let $X \in E$ and $R>0$ be given, set $\mathcal{H}=\mathcal{B}(\epsilon) \cap\left\{(E \cap B(X, R) \times(0, R]\}\right.$, and let $A_{j}$ and $A_{j}^{*}$ be as in the previous argument. The same computations as before apply, except that (3.26) must be replaced with

$$
\begin{aligned}
\Lambda(\mathcal{H}) & \leq C \sum_{j \geq 0} \sum_{x \in A_{j}^{*}}\left\{H^{d}(E(x, t))+\nu(F(x, t))\right\} \\
& \leq C H^{d}\left(\bigcup_{j, x} E(x, t)\right)+C \nu\left(\bigcup_{j, x} F(x, t)\right) .
\end{aligned}
$$

As before we observe that $E(x, t) \cup F(x, t) \subseteq B(X, 5 R)$ for all the relevant $(x, t)$ 's, and so the estimate $\Lambda(\mathcal{H}) \leq C R^{d}$ reduces to (2.1) and (4.9). Thus we conclude that $\mathcal{B}(\epsilon)$ is a Carleson set, as desired. This completes the proof of Theorem 4.13 .

Let us now turn to the proof of Theorem 4.3. Let $E$ and $f$ be as in the statement of the theorem. We would like to apply Theorem 4.13 with $b(x) d x=d \nu$, where $\nu$ is as in Theorem 4.3 , but we need to regularize $f$ first.

Let $\rho>0$ be small, to be chosen soon. Let $\left\{Q_{j}\right\}_{j \in J}$ be an enumeration of the maximal dyadic cubes in $\Omega=\mathbf{R}^{d+1} \backslash E$ such that

$$
\operatorname{diam} Q \leq \rho \operatorname{dist}(Q, E) .
$$

Thus $\left\{Q_{j}\right\}$ provides a fine Whitney decomposition of $\Omega$. Note that $\operatorname{diam} Q_{j} \geq \frac{\rho}{4} \operatorname{dist}\left(Q_{j}, E\right)$ for each $j$, by maximality of these cubes. It is not hard to check that the family $\left\{100 Q_{j}\right\}$ has bounded overlap, with a constant which depends on the dimension but not $\rho$, if $\rho$ is small enough. (The point is that if $100 Q_{i}$ and $100 Q_{j}$ intersect, then the ratio of the diameters of $Q_{i}$ and $Q_{j}$ is bounded by a constant which does not depend on $\rho$, so long as $\rho$ is small enough, because the diameters will each be approximately $\rho$ times the distance to $E$, and the distance to $E$ will be about the same for both $Q_{i}$ and $Q_{j}$.) We can associate to this decomposition a partition of unity $\left\{\phi_{j}\right\}_{j \in J}$ in the usual manner, in such a way that each $\phi_{j}$ is a smooth function such that $0 \leq \phi_{j} \leq 1, \operatorname{supp} \phi_{j} \subset 2 Q_{j},\left|\nabla \phi_{j}\right| \leq C\left(\operatorname{diam} Q_{j}\right)^{-1}$, and $\sum_{j} \phi_{j} \equiv 1$ on $\Omega$.

Define $g$ on $\Omega$ by

$$
g(x)=\sum_{j \in J} \phi_{j}(x)\left\{\frac{1}{\left|Q_{j}\right|} \int_{Q_{j}} f(y) d y\right\} .
$$


Then the Poincaré inequality gives

$$
\frac{1}{\left|Q_{j}\right|} \int_{Q_{j}}|f-g| \leq C \rho \operatorname{dist}\left(Q_{j}, E\right) \frac{1}{\left|Q_{j}\right|} \int_{50 Q_{j}} d \nu(y)
$$

if we require also that $\rho<\frac{1}{100}$. (This condition ensures that if $2 Q_{k}$ touches $Q_{j}$, then $2 Q_{k} \subset 50 Q_{j}$, and both $Q_{k}$ and $Q_{j}$ have roughly the same size.) Given $x \in E$ and $r>0$ we can cover $B(x, r) \backslash E$ with $Q_{j}$ 's and apply (4.34) to get

$$
\begin{aligned}
r^{-d-1} & \int_{B(x, r) \backslash E}|f(u)-g(u)| d u \\
\leq & C r^{-d-1} \int_{B(x, 2 r) \backslash E} \rho \operatorname{dist}(y, E) d \nu(y) \\
\leq & C r^{-d} \int_{B(x, 2 r) \backslash E} \rho d \nu(y) \leq C \rho,
\end{aligned}
$$

since $\nu$ is a Carleson measure with respect to $E$ (as in the statement of Theorem 4.3). We are using here also the fact that $\left\{50 Q_{j}\right\}$ has bounded overlap with a constant that does not depend on $\rho$ (to get that the constant $C$ in (4.35) does not depend on $\rho$ ). Altogether we conclude that if $\rho$ is small enough, then

$$
\inf _{\alpha \in \mathbf{R}} r^{-d-1} \int_{B(x, r) \backslash E}|g(y)-\alpha| d y \geq \frac{a}{2},
$$

where $a$ is as in (4.4).

We are going to need a slightly stronger version of (4.36), in which we integrate only over the set of $y$ 's which are not too close to $E$. To get this stronger version we use the following estimates.

Lemma 4.37. - Let $x \in E$ and $r>0$ be given, and let $M$ denote the mean value of $f$ over $B(x, r)$. Given $t>0$, set $E_{t}=\left\{x \in \mathbf{R}^{d+1}: \operatorname{dist}(x, E)<t\right\}$. Then the Lebesgue measure of $B(x, r) \cap E_{s r}$ is $\leq C s r^{d+1}$, and

$$
\int_{B(x, r) \cap E_{s r}}|f(y)-M| d y \leq C s^{\frac{1}{d+1}} r^{d+1}
$$

for all $s \in(0,1)$, where $C$ depends on $f$ but not on $x, r, s$.

To prove the first part we observe that $B(x, 2 r) \cap E$ can be covered by $\leq C s^{-d}$ balls of radius $2 s r$. This is not hard to do, using the regularity assumption (2.1). The doubles of these balls cover $B(x, r) \cap E_{s r}$, which 
implies the first part of the lemma. The second part of the lemma is an easy consequence of the first part, Hölder's inequality, and the Sobolev-Poincaré estimate

$$
\left\{r^{-d-1} \int_{B(x, r)}|f(y)-M|^{p} d y\right\}^{\frac{1}{p}} \leq r^{-d-1} \int_{B(x, r)} r|\nabla f(y)| d y
$$

where $p=(d+1) / d$. (Actually, one can get a better estimate than (4.38) with more direct arguments.) Of course we are also using our Carleson measure assumption on $|\nabla f|$.

LEMMA 4.40. - There exist $\rho, s, a_{0}>0$ so that for each $x \in E$ and $r>0$ we have

$$
\inf _{\alpha \in \mathbf{R}} r^{-d-1} \int_{B(x, r) \backslash E_{s r}}|g(y)-\alpha| d y \geq a_{0} .
$$

Let $M_{s}$ and $N_{s}$ denote the averages of $f$ and $g$, respectively, over $B(x, r) \backslash E_{s r}$. By standard reasoning it suffices to find a uniform lower bound for

$$
r^{-d-1} \int_{B(x, r) \backslash E_{s r}}\left|g(y)-N_{s}\right| d y
$$

when $\rho$ and $s$ are small enough. However, the difference between this and

$$
r^{-d-1} \int_{B(x, r) \backslash E_{s r}}\left|f(y)-M_{s}\right| d y
$$

is $\leq C \rho$, because of (4.35), and the difference between (4.43) and

$$
r^{-d-1} \int_{B(x, r)}|f(y)-M| d y
$$

is $\leq C s^{\frac{1}{d+1}}$, because of Lemma 4.37. (That is, we can control $\left|M_{s}-M\right|$ using (4.38).) We have a uniform lower bound on (4.44), because of (4.4), and we conclude that there is a uniform lower bound on (4.42) if $\rho$ and $s$ are small enough. This proves the lemma.

Let $\rho, s$, and $a_{0}$ be as in Lemma 4.40, and fixed for the rest of the proof. From now on we can permit our constants to depend on $\rho$. (This was dangerous before, as in (4.36).) 
Notice that $g$ is smooth on $\Omega$. Let $\nabla g$ denote its pointwise gradient on $\Omega$, ignoring any singular distributional part on $E$. Because $\left\{\phi_{j}\right\}$ is a partition of unity we have that $\sum_{j} \nabla \phi_{j} \equiv 0$ on $\Omega$. Using this and the Poincaré inequality it is not hard to show that

$$
\sup _{Q_{j}}|\nabla g(z)| \leq C \frac{1}{\left|Q_{j}\right|} \int_{50 Q_{j}} d \nu(y)
$$

for all $Q_{j}$. (This is similar to (4.34), and it uses the observation that we can control the difference between $\frac{1}{\left|Q_{i}\right|} \int_{Q_{2}} f(y) d y$ and $\frac{1}{\left|Q_{j}\right|} \int_{Q_{j}} f(y) d y$ in terms of the right side of (4.45) when $2 Q_{i}$ touches $Q_{j}$.) Since $\nu$ is a Carleson measure with respect to $E$ (and $\operatorname{diam} Q_{j} \geq \frac{\rho}{4} \operatorname{dist}\left(Q_{j}, E\right)$ ) we get that

$$
|\nabla g(y)| \leq C \operatorname{dist}(y, E)^{-1}
$$

and

$$
d \widetilde{\nu}(y)=|\nabla g(y)| d y \text { is a Carleson measure with respect to } E \text {. }
$$

(Note that $d \widetilde{\nu}(y)$ really lives on $\Omega$, while $\nu$ itself will have a nontrivial singular part on $E$.)

We are now ready to apply Theorem 4.13. Set $b=|\nabla g|$. We have just seen that $b$ satisfies (4.8) and (4.9), and so we only need to verify that there is an $\eta>0$ so that for each $x \in E$ and $t>0$ we can find points $y_{1}, y_{2} \in B(x, t) \backslash E$ which satisfy (4.11) and (4.12). Since $D_{b}\left(y_{1}, y_{2}\right) \geq\left|g\left(y_{1}\right)-g\left(y_{2}\right)\right|$ by definitions, the existence of $\eta, y_{1}$, and $y_{2}$ follows easily from Lemma 4.40 (with $\eta$ given in terms of $s$ and $a_{0}$ ). Thus $E$ satisfies Condition C, and Theorem 4.3 follows from Theorem 4.13.

Remark 4.48. - Theorem 4.3 would not remain valid if we replaced (4.4) with a weaker condition of the type "the jump of $f$ across $E$ is bounded from below". Indeed, if such a condition were sufficient, then we could try to apply it with $f$ taken to be the characteristic function of an open set $U \subset \mathbf{R}^{d+1}$ whose boundary is regular in the sense of (2.1). It is easy to build examples of such sets $U$ whose boundary is not uniformly rectifiable but for which the jump across $\partial U$ is bounded from below. (Basically one should choose $U$ to be a union of many scattered little cubes, as in a suitable approximation to a Cantor set.)

Remark 4.49. - We stated Theorem 4.3 only for real-valued functions $f$, but it is also valid (with the same proof) for vector-valued functions.

Remark 4.50. - Contrary to the situation with the WNPC, we cannot use anything like the slightly more direct argument mentioned in Remark 3.27 
for deriving uniform rectifiability from the hypotheses of Theorems 4.3 or 4.13. Indeed, the next result tells us that the hypotheses of those theorems are actually equivalent to uniform rectifiability, while it is known [Hr] that uniform rectifiability does not imply the existence of big projections or the BPLG condition (see Definitions 3.28 and 3.32), and the approach mentioned in Remark 3.27 necessarily entails those properties. Thus in this case we cannot seem to avoid Theorem 2.14 and the local symmetry condition.

Let us now turn to the converse of Theorem 4.3.

THEOREM 4.51. - Let $E$ be a d-dimensional uniformly rectifiable set in $\mathbf{R}^{d+1}$. Then there is a smooth real-valued function $g$ on $\Omega=\mathbf{R}^{d+1} \backslash E$ and a constant $C>0$ such that

$$
|g(y)| \leq C \text { and }|\nabla g(y)| \leq C \operatorname{dist}(y, E)^{-1} \text { for all } y \in \Omega
$$

$$
|\nabla g(y)| d y \text { is a Carleson measure with respect to } E
$$

and

$$
\inf _{\alpha \in \mathbf{R}} t^{-d-1} \int_{B(x, t) \backslash E}|g(z)-\alpha| d z \geq C^{-1} \text { for all } x \in E \text { and } t>0 \text {. }
$$

The "distributional" version of (4.53) is also true. That is, since $g$ is bounded by (4.52) we can view it as a distribution on all of $\mathbf{R}^{d+1}$, and its distributional gradient is a vector-valued measure whose total variation is a Carleson measure. This strengthening of (4.53) will follow from the proof, but it can also be derived from the boundedness of $g$ and the regularity of $E$. In other words, the singular part of the distributional gradient will be a measure living on $E$ which will be bounded by a constant times Hausdorff measure.

Our construction will also give that $\nabla g=0$ except on a Carleson set in $\Omega$, i.e., a set $H$ such that $\mathbf{1}_{H}(y) \operatorname{dist}(y, E)^{-1} d y$ is a Carleson measure with respect to $E$.

To prove Theorem 4.51 we shall (unfortunately) need to import some results and terminology from [DS3]. Although this will make the proof less pleasant to read, it seems to be the only way to prevent it from becoming too long.

Let $E \subset \mathbf{R}^{d+1}$ be given, as in Theorem 4.51 , and let $\Delta$ be a family of "cubes" on $E$. The elements of $\Delta$ are subsets of $E$ which have the same sort of size and intersection properties as dyadic cubes in $\mathbf{R}^{d}$; see [DS3], Vol. $13, \mathbf{n}^{\circ} 4-1996$. 
p. 53, for details. We shall not use these cubes in a serious way here, but rather as a convenient tool for coding geometric information about $E$.

Because $E$ is uniformly rectifiable, it satisfies the "bilateral weak geometric lemma" (BWGL). This means that for each $\epsilon>0$ and $k>1$ the set

$$
\begin{aligned}
& \mathcal{B}(\epsilon, k)=\{Q \in \Delta: \text { there does not exist a } d \text {-plane } P(Q) \text { such that } \\
& \operatorname{dist}(x, P(Q)) \leq \epsilon \operatorname{diam} Q \text { for all } x \in E \text { which satisfy } \\
& \operatorname{dist}(x, Q) \leq k \operatorname{diam} Q \text { and also } \operatorname{dist}(p, E) \leq \epsilon \operatorname{diam} Q \\
& \text { for all } p \in P(Q) \text { which satisfy } \operatorname{dist}(p, Q) \leq k \operatorname{diam} Q\}
\end{aligned}
$$

(i.e., the set of cubes $Q$ such that $E$ is not well-approximated "bilaterally" by a $d$-plane near $Q$ ) satisfies a Carleson packing condition of the type

$$
\sum_{\substack{Q \in \mathcal{B}(\epsilon, k) \\ Q \subseteq B(x, t)}} H^{d}(Q) \leq C t^{d} \text { for all } x \in E \text { and } t>0
$$

(See p. 32 of [DS3] for the BWGL, and see the discussion around Lemma 3.10 on p. 55 of [DS3] for the packing condition (4.56).) In other words, $E$ is very well approximated by a $d$-plane near most cubes $Q$. We shall choose $\epsilon$ and $k$ later in the argument.

Lemma 3.22 on p. 58 in [DS3] says that there is a "coronization" $(\mathcal{B}, \mathcal{G}, \mathcal{F})$ of $E$ such that $\mathcal{B}(\epsilon, k) \subseteq \mathcal{B}$. The precise definition of a coronization is given on p. 55 of [DS3], and the highlights are these: $\mathcal{B}$ is a set of "bad" cubes in $\Delta$, and it satisfies a Carleson packing condition (like (4.56)); $\mathcal{G}$ is the complement of $\mathcal{B}$ in $\Delta$, the set of "good cubes"; $\mathcal{F}$ is a partition of $\mathcal{G}$ into "stopping-time regions" $S, S \in \mathcal{F}$; each stopping-time region $S$ has a maximal cube $Q(S)$, and the collection of all the maximal cubes $\{Q(S): S \in \mathcal{F}\}$ satisfies a Carleson packing condition (like (4.56)). This Carleson packing condition provides a way of saying that there are not too many of these stopping-time regions. The stopping-time regions satisfy some additional geometric conditions, such as the "connectedness" property that if $Q_{1}$ and $Q_{2}$ lie in one of them, call it $S$, and if $Q$ is a cube which satisfies $Q_{1} \subseteq Q \subseteq Q_{2}$, then $Q$ lies in $S$ also. See [DS3] for more details.

This coronization is useful because it provides a decomposition of the complement of $E$ into a not-too-large family of nice regions for which the geometry of $E$ is pretty trivial. It will then be easy to build approximations to our desired function $g$ in each of these regions, and to combine these approximations in a manner consistent with the claims of Theorem 4.51. To do these things we need some more technical preliminaries. 
Let $A$ be a large number, to be chosen later. We shall call two cubes $Q_{1}, Q_{2} \in \Delta$ "neighbors" if

$$
\begin{aligned}
\operatorname{dist}\left(Q_{1}, Q_{2}\right) & \leq A\left(\operatorname{diam} Q_{1}+\operatorname{diam} Q_{2}\right) \text { and } \\
A^{-1} \operatorname{diam} Q_{1} & \leq \operatorname{diam} Q_{2} \leq A \operatorname{diam} Q_{1} .
\end{aligned}
$$

Because $\mathcal{B}(\epsilon, k) \subseteq \mathcal{B}$ and $\mathcal{B}$ is the complement of $\mathcal{G}$, we can assign to each cube $Q$ in $\mathcal{G}$ a $d$-plane $P(Q)$ with the properties described in (4.55). Thus the part of $E$ not too far from $Q$ lies very close to $P(Q)$, and the part of $P(Q)$ which is not too far from $Q$ must lie very close to $E$. This $d$-plane is not unique, but it turns out that it is determined up to small perturbations, and at any rate we simply pick one that works for each $Q$. Two cubes in $\mathcal{G}$ which are neighbors will have their corresponding $P(Q)$ 's being almost parallel, as in the following.

LeMma 4.58. - If $Q_{1}, Q_{2} \in \mathcal{G}$ are neighbors, and if $k$ is large enough (depending on $A$ ), then $\operatorname{Angle}\left(P\left(Q_{1}\right), P\left(Q_{2}\right)\right) \leq C \epsilon$, where $C$ depends on $A$ but not $\epsilon, Q_{1}$, or $Q_{2}$.

By "Angle $\left(P, P^{\prime}\right)$ " we mean the largest angle between the two $d$-planes $P, P^{\prime}$. Parallel planes have angle 0 , and otherwise the angle measures the extent to which they are not parallel. Lemma 4.58 is the same as Lemma 5.13 in [DS1]. The proof is quite easy; it comes down to the fact that $P\left(Q_{1}\right)$ and $P\left(Q_{2}\right)$ are both approximating the same piece of $E$.

Since each stopping-time region $S \in \mathcal{F}$ is contained in $\mathcal{G}$, we have chosen $d$-planes for all the cubes in every stopping-time region $S$. The next lemma tells us that we can choose unit normals to the $P(Q)$ 's for $Q$ in a fixed stopping-time region $S$ in a coherent way.

LemMA 4.59. - If $\epsilon$ and $k^{-1}$ are chosen small enough (depending on $A$ in particular), then we can choose a unit normal $N(Q)$ to $P(Q)$ for each $Q \in \mathcal{G}$ in such a way that if $S \in \mathcal{F}$ and $Q_{1}, Q_{2} \in S$ are neighbors, then

$$
\left|N\left(Q_{1}\right)-N\left(Q_{2}\right)\right|<\frac{1}{10} \text {. }
$$

This is Lemma 2.22 on p. 105 of [DS3]. The main point is that once we have chosen $N(Q)$ for the maximal cube $Q(S)$ of a stopping-time region $S \in \mathcal{F}$, then we have no choice for the other cubes in $S$, because $P\left(Q_{1}\right)$ and $P\left(Q_{2}\right)$ are always very close when $Q_{1}$ and $Q_{2}$ are neighbors. Note that Lemma 4.58 says that if $Q_{1}$ and $Q_{2}$ are neighbors, then $N\left(Q_{1}\right)$ and $N\left(Q_{2}\right)$ are either almost the same or almost the opposite of each other. Thus, once we have (4.60), we can get a better estimate from Lemma 4.58. 
We now have all the information about $E$ that we need, but we need to convert its coding in terms of cubes in $\Delta$ into information about $\Omega$ (the complement of $E$ ).

Let $\left\{W_{j}\right\}_{j \in J}$ be a Whitney decomposition of $\Omega$. For instance, take the cubes $Q_{j}$ used in the proof of Theorem 4.3 (around (4.32)) and change their names. (Note that the $W_{j}$ 's are supposed to be actual Euclidean cubes, as opposed to the elements of $\Delta$.) In particular we require that $\operatorname{dist}\left(2 W_{j}, E\right) \approx \operatorname{diam} W_{j}$ for all $j$. For each $j \in J$ choose a $Q_{j} \in \Delta$ such that

$$
\operatorname{dist}\left(Q_{j}, W_{j}\right)=\operatorname{dist}\left(W_{j}, E\right)
$$

and

$$
C^{-1} \operatorname{diam} W_{j} \leq \operatorname{diam} Q_{j} \leq C \operatorname{diam} W_{j},
$$

where $C$ does not depend on $j$. ( $C$ can be given in terms of the constants associated to $\Delta$ as in [DS3].)

Define $\alpha_{j} \in\{+1,-1,0\}$ for $j \in J$ as follows. If $Q_{j}$ lies in the bad set $\mathcal{B}$, then set $\alpha_{j}=0$. If $Q_{j} \in \mathcal{G}$, then $W_{j} \subset \mathbf{R}^{d+1} \backslash P\left(Q_{j}\right)$ if $\epsilon$ and $k^{-1}$ are small enough. (This follows from the fact that $\operatorname{dist}\left(W_{j}, E\right) \approx \operatorname{diam} W_{j}$, (4.61), (4.62), and the fact that all elements of $P(Q)$ which are not too far from $Q$ must lie very close to $E$.) In this case we set $\alpha_{j}=+1$ if $W_{j}$ lies on the side of $P\left(Q_{j}\right)$ into which $N\left(Q_{j}\right)$ points, and $\alpha_{j}=-1$ otherwise.

Let $\left\{\phi_{j}\right\}$ be a partition of unity associated to the $W_{j}$ 's in the usual manner (as in [St], p. 168-170). In particular each $\phi_{j}$ satisfies $0 \leq \phi_{j} \leq 1,\left|\nabla \phi_{j}\right| \leq C\left(\operatorname{diam} W_{j}\right)^{-1}$, and $\operatorname{supp} \phi_{j} \subset 2 W_{j}$. Set

$$
g(y)=\sum_{j \in J} \alpha_{j} \phi_{j}(y) \quad \text { for } y \in \Omega .
$$

It is very easy to see that (4.52) holds, using the fact that the cubes $2 W_{j}$ have bounded overlap. We want to show that $\nabla g$ is supported on a Carleson set in $\Omega$. Afterwards we shall verify (4.54) using the fact that our choice of $\alpha_{j}$ 's makes $g$ switch frequently from +1 to -1 across $E$.

Fix a Whitney cube $W_{l}$ and suppose that $\nabla g \neq 0$ somewhere on $W_{l}$. This means that there is a Whitney cube $W_{j}$ such that $2 W_{j} \cap W_{l} \neq \emptyset$ and $\alpha_{j} \neq \alpha_{l}$. If $A$ is sufficiently large, then $Q_{j}$ and $Q_{l}$ must be neighbors. One possibility is that either $Q_{j}$ or $Q_{l}$ lies in the bad set $\mathcal{B}$. Otherwise, if both lie in $\mathcal{G}$, then $2 W_{j} \cup W_{l}$ does not touch $P\left(Q_{j}\right)$ or $P\left(Q_{l}\right)$ if $\epsilon$ and $k^{-1}$ are small enough (for the same reason that $W_{j} \subset \mathbf{R}^{d+1} \backslash P\left(Q_{j}\right)$ when $\left.Q_{j} \in \mathcal{G}\right)$. The fact that $\alpha_{j} \neq \alpha_{l}$ implies that $Q_{j}$ and $Q_{l}$ cannot belong to 
the same stopping-time region in $\mathcal{F}$. This follows from the definition of the $\alpha$ 's, and the compatibility with the orientations given in Lemma 4.59. (It comes down to the fact that $W_{l}$ cannot simultaneously be on opposite sides of the same $d$-plane.) Altogether we get that $Q_{l}$ lies in the set

$$
\begin{aligned}
\mathcal{H}= & \{Q \in \Delta: Q \text { has a neighbor in } \mathcal{B}\} \cup \\
& \{Q \in \mathcal{G}: Q \text { has a neighbor } \\
& \text { in a different stopping-time region than } Q\}
\end{aligned}
$$

Lemma 3.27 and (3.28) on p. 59-60 of [DS3] imply that $\mathcal{H}$ satisfies a Carleson packing condition, i.e.,

$$
\sum_{\substack{Q \in \mathcal{H} \\ Q \subset B(x, t)}} H^{d}(Q) \leq C t^{d} \text { for } x \in E \text { and } t>0 .
$$

Let $H$ denote the set of $l \in J$ such that $Q_{l} \in \mathcal{H}$.

Observe that a given cube $Q \in \mathcal{H}$ cannot arise as a $Q_{l}$ for more than a bounded number of indices $l \in H$. Indeed, if two $W_{l}$ 's have the same $Q_{l} \in \Delta$, then they have to have approximately the same size, and they cannot be too far from each other (compared to their diameters). It is easy to check that any collection of Whitney cubes which have these two properties can have only a bounded number of elements.

This bound on the multiplicity allows us to derive from (4.65) a similar estimate for $H$ and the $W_{l}$ 's:

$$
\sum_{\substack{l \in H \\ W_{l} \cap B(x, t) \neq \emptyset}}\left(\operatorname{diam} W_{l}\right)^{d} \leq C t^{d}
$$

for all $x \in E$ and $t>0$. We are using here the observation that if $W_{l}$ intersects $B(x, t)$, then $\operatorname{diam} W_{l} \approx \operatorname{dist}\left(W_{l}, E\right) \leq t$, and so $Q_{l} \subset B(x, C t)$. Remember also that $H^{d}(Q) \geq C^{-1}(\operatorname{diamQ})^{d}$ for all cubes $Q \in \Delta$.

Let $\mathcal{V}$ denote the union of all the $W_{l}$ 's on which $\nabla g$ does not vanish identically. Thus $\mathcal{V} \subseteq \cup_{l \in H} W_{l}$, and (4.66) implies that

$$
\int_{\mathcal{V} \cap B(x, t)} \operatorname{dist}(y, E)^{-1} d y \leq C t^{d}
$$

for all $x \in E$ and $t>0$. This is the Carleson measure estimate on $\mathcal{V}$ that we wanted, and it implies (4.53) immediately (with the help of (4.52)). 
We are left with the task of verifying (4.54). The point is that we defined $g$ so that if $Q \in \mathcal{G}$, then, near $Q, g$ should be about +1 on one side of $P(Q)$ and about -1 on the other. More precisely, let us show that for each $x \in E$ and each $t>0$ there exist Whitney cubes $W_{l}$ and $W_{m}$ such that

$$
\begin{gathered}
W_{l} \cup W_{m} \subseteq B(x, t), \\
\operatorname{diam} W_{l} \geq C^{-1} t \text { and } \operatorname{diam} W_{m} \geq C^{-1} t, \text { and } \\
g(y) \equiv 1 \text { on } W_{l} \text { and } g(y) \equiv-1 \text { on } W_{m} .
\end{gathered}
$$

It is easy to see that (4.54) will follow once we have established this fact.

Let $x \in E$ and $t>0$ be given. Because of (4.65), we can find a cube $Q \in \Delta$ such that $Q \subset B\left(x, \frac{t}{2}\right), \operatorname{diam} Q \geq \frac{t}{C^{\prime}}$, and $Q \notin \mathcal{H}$. (Indeed, the sum of the measures $H^{d}(Q)$ of the cubes $Q$ that satisfy only the first two conditions and not the third grows like $t^{d} \log C^{\prime}$ as $C^{\prime} \rightarrow \infty$, and if all these cubes were in $\mathcal{H}$ then this would contradict (4.65) when $C^{\prime}$ is sufficiently large.) If the neighborly constant $A$ in (4.57) is chosen large enough, then we can find Whitney cubes $W_{l}$ and $W_{m}$ (one on each side of $P(Q)$ ) that satisfy the desired conditions (4.68), (4.69), and (4.70).

Thus we can choose the constant $A$, and then $\epsilon$ and $k$, in such a way that the function $g$ that we have defined satisfies all the required properties. This completes the proof of Theorem 4.51. This proof also gives the following more precise converse to Theorem 4.13.

THEOREM 4.71. - Let $E$ be a d-dimensional uniformly rectifiable set in $\mathbf{R}^{d+1}$. Then $E$ satisfies Condition $C$, and we can even choose $b(y)$ so that $0 \leq b(y) \leq \operatorname{dist}(y, E)^{-1} \mathbf{1}_{\mathcal{V}}(y)$, where $\mathcal{V} \subset \mathbf{R}^{d+1} \backslash E$ satisfies the Carleson condition

$$
\int_{\mathcal{V} \cap B(x, t)} \operatorname{dist}(y, E)^{-1} d y \leq C t^{d} \quad \text { for all } \quad x \in E \text { and } t>0 .
$$

This follows from the construction above. More precisely, we take $\mathcal{V}$ and $g$ to be as defined in the preceding argument, and we set $b=C^{-1}|\nabla g|$ for a suitably large constant $C$. Then we have already seen that (4.8), (4.9), and (4.72) are satisfied, and we need only check that we can find points $y_{1}$ and $y_{2}$ as in (4.11) and (4.12). This follows from the existence of the Whitney cubes $W_{l}$ and $W_{m}$ as in (4.68)-(4.70) and the fact that $D_{b}\left(y_{1}, y_{2}\right) \geq C^{-1}\left|g\left(y_{1}\right)-g\left(y_{2}\right)\right|$.

Remark 4.73. - It is interesting to compare the various situations in which uniformly rectifiable sets arise as singular sets of functions. If $d=1$, so that we can identify $\mathbf{R}^{d+1}$ with the complex plane, then we can build 
holomorphic functions on the complement of $E$ with large jump across $E$ by taking the Cauchy integral of suitable suitable measures on $E$ (like Hausdorff measure). A similar construction in higher dimensions produces Riesz systems or Clifford-holomorphic functions with a large jump. These holomorphic functions satisfy weaker quadratic versions of the gradient estimate (4.53) (see (2.39) in Definition 2.38 on p. 44 of [DS3]), but they have the advantage of being holomorphic. In other words, in this section we are making the trade-off of giving up holomorphicity in exchange for stronger gradient estimates. (Compare with Theorem 3.4 on p. 240 and Exercise 13 on p. 275 of [Ga].) In the next section we shall deal with functions which satisfy stronger Carleson measure conditions on their gradients but which have weaker singularities.

\section{MINIMIZERS OF THE MUMFORD-SHAH FUNCTIONAL AND ITS VARIANTS}

Let $\Omega \subset \mathbf{R}^{d+1}$ be a nice domain. To simplify the exposition we assume for the time being that $\Omega$ is a cube or a ball in $\mathbf{R}^{d+1}$, but we shall discuss general conditions on $\Omega$ which will suffice for our purposes in the remarks at the end of the section. Also let $1<p<\infty$ and $g \in L^{\infty}(\Omega)$ be given. We want to consider the functional

$$
J(u, K)=\int_{\Omega \backslash K}|u-g|^{2}+\int_{\Omega \backslash K}|\nabla u|^{p}+H^{d}(K),
$$

where $H^{d}$ denotes $d$-dimensional Hausdorff measure, and the competitors $(u, K)$ are required to satisfy the following conditions:

(5.2) the set $K$ is (relatively) closed in $\Omega$ and $H^{d}(K)<\infty$;

(5.3) $u$ is a function defined on $\Omega \backslash K$ whose distributional gradient $\nabla u$ lies in $L^{p}(\Omega \backslash K)$ (so that $u \in W^{1, p}(\Omega \backslash K)$ ).

(The integrals in (5.1) are taken with respect to ordinary Lebesgue measure.)

This is a generalization of the Mumford-Shah functional introduced in [MuS] (which corresponds to $p=2$ and $d=1$ ). We are allowing an exponent $p \neq 2$ in the gradient term because it is not clear whether powers $p>2$ (like $p=d+1$ ) will be more natural in higher dimensions. For the purposes of this paper the choice of exponent will make no difference. We could also change the power 2 in the first integral without ill effect, but that is less likely to have substantial consequences for the properties of 
$J(u, K)$. Also, we could have allowed multiplicative constants to modify the relative weighting of the three terms in (5.1) without seriously affecting the arguments to follow.

There is a very general existence theorem for minimizers of a large class of functionals including $J(u, K)$ given in [Am], but this result requires also a larger class of competitors. It turns out that for $J(u, K)$ these generalized minimizers are equivalent to competitors which satisfy (5.2) and (5.3). This was proved in [DCL] for the case $p=2$. Unfortunately the $p \neq 2$ case does not seem to be stated anywhere in exactly this way, but the paper [CL2] deals with the more complicated case of maps into spheres, and their arguments apply to this situation as well. The proofs of these existence results also imply that the singular sets of minimizers are rectifiable.

See $[\mathrm{MoS}]$ for more information about the Mumford-Shah functional, particularly in connection with image segmentation and the properties of its minimizers. (See also [DS5] for the latter.)

Let us call a competitor $(u, K)$ irreducible if there is not a proper closed subset $\widetilde{K}$ of $K$ such that $u$ extends to a $C^{1}$ function on $\Omega \backslash \widetilde{K}$. It is not hard to check that any minimizer $(u, K)$ can be replaced with an irreducible minimizer (also called "an essential minimizing pair" in Remark 4.6 of [CL2]). This is useful, since it excludes extraneous isolated points, etc., from $K$.

THEOREM 5.4. - Let $\Omega, g$, and $J(\cdot, \cdot)$ be as above, and let $(u, K)$ be an irreducible minimizer for $J(\cdot, \cdot)$. Then $K$ is contained in a d-dimensional uniformly rectifiable set.

As in Definition 2.9, the conclusion means that there is an $A_{1}$-weight $\omega$ and an $\omega$-regular mapping $z: \mathbf{R}^{d} \rightarrow \mathbf{R}^{d+2}$ such that $K \subseteq z\left(\mathbf{R}^{d}\right)$. Of course the part of $z\left(\mathbf{R}^{d}\right)$ which is far from $\Omega$ does not really matter here.

The proof of Theorem 5.4 will show that the uniform rectifiability constants (i.e., the constants in (2.5)-(2.8)) can be controlled by a function of $\Omega, p, d$, and $\|g\|_{\infty}$ only. Some variants of Theorem 5.4 will be described in the Remarks 5.47 and 5.49 below.

When $d=1$ this theorem implies that the singular set $K$ of any irreducible minimizer is contained in a regular curve. This was already proved in [DS4] (at least when $p=2$, but the method also works in general). The argument here will be somewhat different, and more successful in higher dimensions.

A crucial ingredient in the proof of Theorem 5.4 is the "Elimination Lemma": under the assumptions of the theorem, there is a constant $C_{0}$ (depending on $p, d$, and $\|g\|_{\infty}$ ) such that

$$
H^{d}(K \cap B(x, r)) \geq C_{0}^{-1} r^{d} \quad \text { for all } x \in K \text { and } 0<r<\operatorname{dist}(x, \Omega) .
$$


When $d=1$ and $p=2$ this was proved in [DMS]. When $d>1$ and $p=2$ this follows from Remark 3.13 in [CL1], although it is not stated explicitly in this form. A more general result is given in Lemma 4.9 [CL2], but unfortunately that result is stated only for the more complicated situation where the mappings take values in spheres (instead of simply $\mathbf{R}$ ). Although it is not stated explicitly in [CL2], their methods apply also to the real-valued case.

Let us now prove the theorem. Let $(u, K)$ be an irreducible minimizer for $J(\cdot, \cdot)$. Our plan is to show that $K$ satisfies a local version of the WNPC (Definition 3.4). To do this we shall need some preliminary estimates, especially for $\nabla u$.

LEMMA 5.6. - There is a constant $C$ (depending on $\|g\|_{\infty}$ ) such that

$$
H^{d}(K \cap B(x, r)) \leq C r^{d}
$$

and

$$
\int_{\Omega \cap B(x, r) \backslash K}|\nabla u|^{p} \leq C r^{d}
$$

for all $x \in K$ and $0<r \leq 1$.

This follows from a simple, brutal, and well-known truncation argument. If we replace $K \cap B(x, r)$ with $\partial B(x, r)$ and $u$ by 0 on $B(x, r)$, then we get a new competitor $(\widetilde{u}, \widetilde{K})$ such that

$$
\begin{aligned}
\int_{\Omega \backslash \widetilde{K}}|\widetilde{u}-g|^{2} & \leq \int_{\Omega \backslash \widetilde{K}}|u-g|^{2}+\|g\|_{\infty}^{2}|B(x, r)| \\
& \leq \int_{\Omega \backslash \widetilde{K}}|u-g|^{2}+C r^{d}, \\
\int_{\Omega \backslash \widetilde{K}}|\nabla \widetilde{u}|^{p} & =\int_{\Omega \backslash \widetilde{K}}|\nabla u|^{p}-\int_{\Omega \cap B(x, r) \backslash \widetilde{K}}|\nabla u|^{p},
\end{aligned}
$$

and

$$
H^{d}(\widetilde{K}) \leq H^{d}(K)-H^{d}(K \cap B(x, r))+H^{d}(\partial B(x, r))
$$

The estimates (5.7) and (5.8) follow from these inequalities and the fact that $J(u, K) \leq J(\widetilde{u}, \widetilde{K})$, since $(u, K)$ is a minimizer. 
Note that the mass bounds (5.5) and (5.8) imply that $K$ is "locally" regular (in the sense of (2.1)).

For the next estimate fix any $q \in(1, p)$ and set

$$
\omega_{q}(x, t)=t\left\{\frac{1}{t^{d+1}} \int_{B(x, t) \backslash K}|\nabla u|^{q}\right\}^{\frac{p}{q}}
$$

for all pairs $(x, t) \in \Delta=\{(y, s) \in K \times(0,1]: B(y, s) \subseteq \Omega\}$. (This set $\Delta$ is a local version of the set $K \times \mathbf{R}_{+}$which we have used so much before, but it is unrelated to the $\Delta$ which we used in the previous section.) Hölder's inequality (or Jensen's) implies that $\omega_{q}(x, t) \leq C \omega_{p}(x, t)$, which is itself $\leq C$ by (5.8). The reason for taking $q<p$ is that we can do much better than this.

Lemma 5.13. - The function $\omega_{q}(x, t)$ satisfies the local Carleson measure estimate

$$
\iint_{(x, t) \in(K \cap B(X, R)) \times(0, R]} \omega_{q}(x, t) d \Lambda(x, t) \leq C R^{d}
$$

for some $C>0$ and all $X \in K$ and $0<R \leq \min \left(1, \frac{1}{3} \operatorname{dist}(X, \partial \Omega)\right)$, where $d \Lambda$ denotes the measure $t^{-1} d H^{d}(x) d t$ on $\Delta$.

This lemma can be verified without much pain using Hölder's inequality, Fubini's theorem, the mass bounds (5.7) and (5.5), and also (5.8). We shall omit the proof because it is the same as the proof of Proposition 4.5 in [DS4], modulo cosmetic changes pertaining to the dimension and the more general exponents permitted here. However, as a demonstration of our solidarity with the reader, let us indicate the main points. Given $\eta>0$, consider the quantity

$$
t\left\{\frac{1}{t^{d+1}} \int_{B(x, t) \backslash K}|\nabla u(y)|^{p}\left(t^{-1} \operatorname{dist}(y, K)\right)^{\eta} d y\right\} .
$$

Like $\omega_{q}(x, t)$, this is an average of $|\nabla u|$ which is smaller than $\omega_{p}(x, t)$. The analogue of Lemma 5.13 for this quantity instead of $\omega_{q}(x, t)$ is much easier to prove, using Fubini and a technical lemma to reduce to (5.8). Lemma 5.13 itself can be reduced to this simpler variant when $\eta$ is small enough using Hölder's inequality and another technical lemma.

With these preliminary estimates out of the way let us now show that $K$ satisfies a local version of the WNPC. Let $k>1$ and $M>0$ be given and fixed. We may as well assume that $k>10$. Suppose that $(x, t) \in K \times(0,1]$ satisfies $B(x, k t) \subset \Omega$ and $(x, t) \in \mathcal{B}\left(C_{1}, k, M\right)$, where $\mathcal{B}\left(C_{1}, k, M\right)$ is as 
defined at the beginning of Section 3 (see (3.3)), but with $E$ replaced by $K$, and where $C_{1}$ is large, to be chosen soon. We want to show that these properties imply that $\omega_{q}(x, k t)$ is not too small, so that the set of these $(x, t)$ 's will be controlled by Lemma 5.13 . We shall only be able to do this for $q$ close to $p$, but that will be adequate for our purposes.

By definition of $\mathcal{B}\left(C_{1}, k, M\right)$, we have that (3.3) holds (with $E=K$ ) for all $f \in C^{\infty}(B(x, k t) \backslash K)$. A standard limiting argument implies that (3.3) is also true for any $f \in W^{1, p}(B(x, k t) \backslash K)$, and for the function $u$ in particular. Hence there is a real number $\alpha$ such that

$$
\begin{aligned}
\left|m_{B_{1}} u-\alpha\right| & \leq M t^{-d} \int_{B(x, k t) \backslash K}|\nabla u| \\
& \leq C t^{1-\frac{1}{p}} \omega_{1}(x, k t)^{\frac{1}{p}} \leq C t^{1-\frac{1}{p}} \omega_{q}(x, k t)^{\frac{1}{p}}
\end{aligned}
$$

for all balls $B_{1} \subset B(x, t) \backslash K$ with radius $\geq C_{1}^{-1} t$. The constants $C$ in (5.15) depend on $k$ and $M$, but we do not mind. We may as well require also that $|\alpha| \leq\|u\|_{\infty}$.

The basic idea in the argument that follows is quite simple. If $\omega_{q}(x, k t)$ is very small, then (5.15) says that $u$ oscillates very little on $B(x, t)$. We then modify $(u, K)$ in such a way as to remove a substantial portion of $K$ inside $B(x, t)$, and hence reduce the $H^{d}(K)$ term in (5.1) substantially, while only adding a small amount to the other two terms in $J(u, K)$. This will contradict the minimality of $J(u, K)$. In making this modification we cannot operate too brutally on $B(x, t)$ itself; we need to choose first a slightly smaller ball $B(x, r)$ whose boundary has small intersection with $K$ and other nice properties. Once we choose this ball we shall then modify $K$ and $u$.

Let us now choose the ball $B(x, r) \subset B(x, t)$ such that $K \cap \partial B(x, r)$ is reasonably small, etc. Let $\eta$ be a small positive number ( $\eta<\frac{1}{10}$ anyway), to be chosen soon, and cover $K \cap B(x, t)$ by balls $D_{i}, i \in I$, which are centered on $K \cap B(x, t)$ and which all have radius $\eta t$. We can do this with $\leq C \eta^{-d}$ balls, because of the mass bounds (5.5) and (5.7). We claim that we can find a radius $r$ such that

$$
\frac{t}{2} \leq r \leq \frac{3 t}{4}
$$

and

(5.17) the set $I_{0}$ of indices $i \in I$ such that $2 \bar{D}_{i}$ touches $\partial B(x, r)$ has $\leq C \eta^{1-d}$ elements. 
This is easy to see, because we can find more than $(C \eta)^{-1}$ choices of radii $r$ which satisfy (5.16) and for which the corresponding sets of indices $I_{0}$ are disjoint. We can even choose $r$ so that it also satisfies

$$
\begin{aligned}
\int_{\partial B(x, r) \backslash K}|\nabla u|^{q} d H^{d} & \leq C t^{-1} \int_{B(x, t) \backslash K}|\nabla u|^{q} d H^{d+1} \\
& \leq C t^{d}\left(\frac{\omega_{q}(x, t)}{t}\right)^{\frac{q}{p}}
\end{aligned}
$$

because of the usual Fubini theorem argument. (If we choose $C$ large enough, then at least half the $r$ 's in the range (5.16) satisfy (5.18), and that is enough so that at least one of them also satisfies (5.17).) Fix such a radius $r$.

Next we want to modify $(u, K)$ on $\bar{B}(x, r)$ to get a new competitor $(\widetilde{u}, \widetilde{K})$. We start with $K$. Set

$$
Z=\partial B(x, r) \cap\left(\bigcup_{i \in I_{0}} 2 \bar{D}_{i}\right)
$$

and

$$
\widetilde{K}=(K \cup Z) \backslash(B(x, r) \cap K) .
$$

Notice that $\widetilde{K}$ is closed, since $K \cap \partial B(x, r) \subset Z$, and also that

$$
\begin{aligned}
H^{d}(\tilde{K}) & \leq H^{d}(K)+H^{d}(Z)-H^{d}(K \cap B(x, r)) \\
& \leq H^{d}(K)+C \eta t^{d}-C_{0}^{-1} r^{d} .
\end{aligned}
$$

This uses the bound in (5.17) on the number of elements of $I_{0}$ and also (5.5). We now choose $\eta$ to be so small that (5.21) implies

$$
H^{d}(\widetilde{K}) \leq H^{d}(K)-\left(2^{d+1} C_{0}\right)^{-1} t^{d} .
$$

We can do this in such a way that $\eta$ does not depend on $C_{1}$ (which will be chosen later), $k$, or $M$ (let alone $x$ or $t$ ).

Our next task is to modify $u$ to get a new function $\widetilde{u}$ on $\Omega \backslash \widetilde{K}$. We shall leave $u$ alone off $\bar{B}(x, r)$, but we shall make a substantial change inside $B(x, r)$ in order to get rid of the singularities of $u$ there. We shall first build a function $h$ on $\partial B(x, r)$ which agrees with $u$ off $Z$, and then extend $h$ to $B(x, r)$ in a nice way.

In the following we shall need to work with the restriction of $u$ to $\partial B(x, r) \backslash Z$. Of course this does not quite make sense for merely a function 
in $W^{1, p}$. The definition of $u$ implies that it satisfies a PDE off $K$ whose leading term is the $p$-Laplacian of $u$, and we could use the well-known regularity theory for such things to get better information about $u$, but overall it is probably simpler to employ the usual technicalities about Sobolev spaces. Since $u \in W^{1, p}(\Omega \backslash K), u$ has a "trace" on $\partial B(x, r) \backslash K$ which is locally in $L^{p}$ and which we also denote by $u$. We can even be careful in choosing $r$ and get that this trace lies in $W^{1, q}(\partial B(x, r) \backslash K)$, and that the tangential part of the gradient of this trace is controlled by (5.18). These technical points are quite standard, and we shall be a little sloppy in referring to them. We might even use the expression "the restriction of $u$ to $\partial B(x, r) \backslash K$ " instead of saying "trace".

Let $\phi$ be a smooth function on $\mathbf{R}^{d+1}$ such that $0 \leq \phi \leq 1$,

$$
\begin{gathered}
\phi(z)=0 \quad \text { when } \operatorname{dist}(z, K) \leq \frac{3 \eta t}{4} \\
\phi(z)=1 \quad \text { when } \operatorname{dist}(z, K) \geq \eta t
\end{gathered}
$$

and

$$
|\nabla \phi(z)| \leq C \eta^{-1} t^{-1}
$$

The construction of such a function is a standard exercise.

Define a function $h$ on $\partial B(x, r)$ by

$$
h(z)=(u(z)-\alpha) \phi(z)+\alpha,
$$

where $\alpha$ is the same number as in (5.15). Strictly speaking, we are using here the trace of $u$ on $\partial B(x, r) \backslash K$, and we interpret (5.26) to mean that $h=\alpha$ on a neighborhood of $\partial B(x, r) \cap K$, even though $u$ is not defined on $K$. By (5.24) and the definition of $Z$ we have that

$$
h(z)=u(z) \quad \text { on } \quad z \in \partial B(x, r) \backslash Z .
$$

Notice also that $h \in W^{1, q}(\partial B(x, r))$.

Take $C_{1}$ to be $(10 \eta)^{-1}$. With this choice we claim that

$$
\int_{\partial B(x, r)}|\nabla h|^{q} d H^{d} \leq C t^{d}\left(\frac{\omega_{q}(x, k t)}{t}\right)^{\frac{q}{p}},
$$

where the constant $C$ may depend on $\eta, k$, and $M$.

Vol. 13, n 4-1996. 
To prove this claim we begin by observing that $|\nabla h| \leq|\nabla u| \mathbf{1}_{\partial B(x, r) \backslash K}+$ $|u-\alpha||\nabla \phi|$, so that the left side of (5.28) is at most a constant times

$$
\int_{\partial B(x, r) \backslash K}|\nabla u|^{q} d H^{d}+\int_{Z_{0}}\left[C \eta^{-1} t^{-1}|u-\alpha|\right]^{q} d H^{d},
$$

where $Z_{0}=\left\{z \in \partial B(x, r): \frac{3}{4} \eta t \leq \operatorname{dist}(z, K) \leq \eta t\right\}$. The first integral is at most $C t^{d}\left(t^{-1} \omega_{q}(x, k t)\right)^{\frac{q}{p}}$ by (5.18), and so we only need to estimate the second one.

Given $z \in Z_{0}$, let $B_{1}=B_{1}(z)$ denote the ball $B\left(z, \frac{\eta t}{10}\right)$. We shall need the estimate

$$
\int_{B_{1} \cap \partial B(x, r)}\left|u-m_{B_{1}} u\right|^{q} d H^{d} \leq C t^{q-1} \int_{B_{1}}|\nabla u|^{q},
$$

where $C$ depends on $\eta$. This inequality is basically well known, but let us quickly sketch a proof. It is helpful to take $B_{2}$ to be a ball contained in $B_{1}$ such that $2 B_{2}$ does not intersect $\partial B(x, r)$ and the radius of $B_{2}$ is at least $10^{-3} \eta t$. To prove (5.30) it suffices to show that

$$
\int_{B_{1} \cap \partial B(x, r)}\left|u-m_{B_{2}} u\right|^{q} d H^{d} \leq C t^{q-1} \int_{B_{1}}|\nabla u|^{q},
$$

since the usual Poincaré inequality will control $\left|m_{B_{1}} u-m_{B_{2}} u\right|$. Suppose for the moment that $u$ is $C^{1}$. Then for each $y, z \in B_{1}$ we can estimate $|u(y)-u(z)|$ in terms of the integral of $|\nabla u|$ over the segment that joins them, and then it is an simple matter to get (5.31) by averaging over $y \in B_{1} \cap \partial B(x, r)$ and $z \in B_{2}$. The general case can be reduced to the estimate for $C^{1}$ functions by standard approximation arguments. This proves (5.30).

Now let us use (5.30) to prove (5.28). With $B_{1}(z)$ as above, notice that we chose $C_{1}$ in such a way that $B_{1}$ satisfies (5.15). We can cover $Z_{0}$ with a family of balls like $B_{1}$ with bounded overlap and then use (5.15) and (5.30) on each ball and sum to get

$$
\begin{aligned}
\int_{Z_{0}}|u-\alpha|^{q} d H^{d} & \leq C t^{q-1} \int_{B(x, 2 r)}|\nabla u|^{q}+C t^{d}\left\{t^{1-\frac{1}{p}} \omega_{q}(x, k t)^{\frac{1}{p}}\right\}^{q} \\
& \leq C t^{q+d}\left(\frac{\omega_{q}(x, k t)}{t}\right)^{\frac{q}{p}}
\end{aligned}
$$

This implies the estimate that we need on the second term in (5.29) to get (5.28), and so our claim is established. 
We now define $\widetilde{u}$ on $B(x, r)$ to be the harmonic extension of the function $h$, i.e., the Poisson extension of $h$. If we choose $q$ to be sufficiently close to $p$ (one can easily compute a suitable range), then $\widetilde{u}$ lies in $W^{1, p}(B(x, r))$ and satisfies

$$
\int_{B(x, r)}|\nabla \widetilde{u}|^{p} \leq C t^{d+1} t^{\frac{-d_{p}}{q}}\left\{\int_{\partial B(x, r)}|\nabla h|^{q} d H^{d}\right\}^{\frac{p}{q}} .
$$

(This is pretty standard and not hard to check.) Combining this with (5.28) gives

$$
\int_{B(x, r)}|\nabla \widetilde{u}|^{p} \leq C t^{d} \omega_{q}(x, k t) .
$$

Set $\widetilde{u}=u$ on the complement of $B(x, r) \cup Z$. Because of (5.27), $\widetilde{u} \in W^{1, p}(\Omega \backslash \widetilde{K})$. The main point here is that we have chosen $\widetilde{u}$ in such a way that it does not jump across $\partial B(x, r) \backslash Z$, even though it will jump across $Z$, and so the distributional gradient of $\widetilde{u}$ will be locally integrable around $\partial B(x, r) \backslash Z$. It is easy to check that $(\widetilde{u}, \widetilde{K})$ is an acceptable competitor for $J(\cdot, \cdot)$, and so $J(u, K) \leq J(\widetilde{u}, \widetilde{K})$. We want to use this inequality to get a lower bound for $\omega_{q}(x, k t)$. The idea is that if $\omega_{q}(x, k t)$ is very small, then the amount of additional energy that $\widetilde{u}$ has is small compared to the amount of $K$ that we removed to get $\widetilde{K}$, in contradiction to the minimality of $J(u, K)$.

To estimate $J(\widetilde{u}, \widetilde{K})$ we begin by observing that $\|u\|_{\infty} \leq\|g\|_{\infty}$. Indeed, if this were not true, then we could reduce $J(u, K)$ by truncating $u$, and contradict the minimality of $(u, K)$. It is not hard to check that $\|h\|_{\infty} \leq\|u\|_{\infty}$, by the definition (5.26) of $h$, and hence that $\|\widetilde{u}\|_{\infty} \leq\|u\|_{\infty}$, by definition of $\widetilde{u}$. Thus $\|\widetilde{u}\|_{\infty} \leq\|g\|_{\infty}$. This and the definition of $\widetilde{u}$ imply that

$$
\int_{\Omega \backslash \widetilde{K}}|\widetilde{u}-g|^{2} \leq \int_{\Omega \backslash K}|u-g|^{2}+C_{2} t^{d+1}\|g\|_{\infty}^{2}
$$

where the constant $C_{2}$ can be taken to be 4 times the volume of the unit ball in $\mathbf{R}^{d+1}$.

From (5.35), (5.34), and (5.22) we obtain that

$$
J(\widetilde{u}, \widetilde{K}) \leq J(u, K)-\left(2^{d+1} C_{0}\right)^{-1} t^{d}+C t^{d} \omega_{q}(x, k t)+C_{2} t^{d+1}\|g\|_{\infty}^{2} .
$$

This implies that

$$
\omega_{q}(x, k t) \geq C(k, M)^{-1}
$$

Vol. $13, \mathrm{n}^{\circ} 4-1996$. 
when $t \leq t_{0}=\left[2^{d+2} C_{0} C_{2}\|g\|_{\infty}^{2}\right]^{-1}$.

Altogether now we have shown that there is a constant $C_{1}$ such that for all $k>10$ and all $M>0$ there exists a constant $C(k, M)$ so that (5.37) holds whenever $x \in K$ and $t \in\left(0, t_{0}\right]$ satisfy $B(x, k t) \subseteq \Omega$ and $(x, t) \in \mathcal{B}\left(C_{1}, k, M\right)$. Therefore, by Lemma 5.13,

$$
\begin{aligned}
\mathcal{B}=\left\{(x, t) \in K \times\left(0, t_{0}\right]: \operatorname{dist}(x, \partial \Omega)<k t\right. \\
\left.\quad \text { and }(x, t) \in \mathcal{B}\left(C_{1}, k, M\right)\right\} \text { is a "local Carleson set". }
\end{aligned}
$$

This means that

$$
\begin{aligned}
& \Lambda(\mathcal{B} \cap([K \cap B(X, R)] \times(0, R])) \leq C R^{d} \\
& \text { for all } X \in K \text { and } 0<R \leq \min \left(1, \frac{1}{3} \operatorname{dist}(X, \partial \Omega)\right),
\end{aligned}
$$

where $\Lambda$ is as in Lemma 5.13 .

We are now almost finished with the proof of Theorem 5.4, but before we finish it off let us specify some general conditions on $\Omega$ under which the rest of the proof will work. (The argument so far works for any open set.) The simplest conditions for us to use at the moment are the following:

$$
\Omega \text { is bounded, }
$$

and

$$
\begin{aligned}
& \text { there is a } d \text {-dimensional regular set } E \\
& \text { which satisfies the WNPC condition and contains } \partial \Omega \text {. }
\end{aligned}
$$

Under these conditions we shall show that $F=K \cup E$ is a regular set which satisfies the WNPC. Theorem 5.4 will then follow from Theorem 3.5.

Let us first check that $F$ is regular. It is clearly closed. We have

$$
H^{d}(F \cap B(x, r)) \leq C r^{d}
$$

because of (5.7), (5.40), and the corresponding estimate for $E$. Next,

$$
H^{d}(F \cap B(x, r)) \geq C^{-1} r^{d}
$$

holds trivially when $x \in E$, and also when $r \geq 2 \operatorname{dist}(x, \partial \Omega)$, because $E$ is regular. When $x \in K$ and $r<2 \operatorname{dist}(x, \partial \Omega)$ we simply use (5.5). Thus $F$ is regular. 
Now we want to check that $F$ satisfies the WNPC. Denote by $\mathcal{B}_{E}(C, k, M)$ the subset of $E \times \mathbf{R}_{+}$that was defined around (3.3), and let $\mathcal{B}_{F}(C, k, M)$ denote the corresponding object for $F$. Our assumption (5.41) implies that there is a constant $C_{E}$ such that $\mathcal{B}_{E}\left(C_{E}, k, M\right)$ is a Carleson set in $E \times \mathbf{R}_{+}$for all $k>1$ and $M>0$.

LEMmA 5.44. - If $C_{F}=C_{3} \max \left(C_{1}, C_{E}\right)$, where $C_{3}$ is a sufficiently large constant (which depends only on $d$ and the regularity constants for $E$ and $F$ ), then $\mathcal{B}_{F}\left(C_{F}, k, M\right)$ is a Carleson set in $F \times \mathbf{R}_{+}$for all choices of $k>1$ and $M>0$.

Of course this says that $F$ satisfies the WNPC.

Let $k>1$ and $M>0$ be given. We may as well assume that $k>10$. Define $\mathcal{A}_{1}, \mathcal{A}_{2}, \mathcal{A}_{3} \subset F \times \mathbf{R}_{+}$as follows:

$$
\begin{gathered}
\mathcal{A}_{1}=\left\{(x, t) \in \mathcal{B}_{F}\left(C_{F}, k, M\right): t \geq 10 \operatorname{dist}(x, E)\right\} ; \\
\mathcal{A}_{2}=\left\{(x, t) \in \mathcal{B}_{F}\left(C_{F}, k, M\right): x \in K, t \leq t_{0}, \text { and } k t<\operatorname{dist}(x, \partial \Omega)\right\} ; \\
\mathcal{A}_{3}=\left\{(x, t) \in \mathcal{B}_{F}\left(C_{F}, k, M\right):\right. \\
\left.x \in K \text { and } \min \left(t_{0}, k^{-1} \operatorname{dist}(x, \partial \Omega)\right) \leq t \leq 10 \operatorname{dist}(x, E)\right\} .
\end{gathered}
$$

It suffices to show that each of these is a Carleson set.

Let us begin with $\mathcal{A}_{1}$. Let $(x, t) \in \mathcal{A}_{1}$ be given. We claim that each pair $(y, s)$ with $y \in E \cap B\left(x, \frac{t}{5}\right)$ and $\frac{t}{2} \leq s \leq \frac{2 t}{3}$, say, lies in $\mathcal{B}_{E}\left(C_{E}, 3 k, M^{\prime}\right)$ for some $M^{\prime}$ which can be computed in terms of $M, k, C_{3}$, and $d$. To prove this suppose that $(y, s)$ satisfies the preceding conditions but that $(y, s) \notin \mathcal{B}_{E}\left(C_{E}, 3 k, M^{\prime}\right)$. Then there is a function $f$ in $C^{\infty}(B(y, 3 k s) \backslash E)$ and balls $B_{1}, B_{2} \subset B(y, s) \backslash E$ with radii $\geq s C_{E}^{-1}$ such that

$$
\left|m_{B_{1}} f-m_{B_{2}} f\right| \geq M^{\prime} s^{-d} \int_{B(y, 3 k s) \backslash E}|\nabla f| .
$$

Our restrictions on $y$ and $s$ imply that $f \in C^{\infty}(B(x, k t) \backslash F)$ and that the balls $B_{1}, B_{2}$ are also contained in $B(x, t)$. However, it is possible that these balls will intersect $K$ and hence $F$. To get around this difficulty we observe that if $C_{3}$ is large enough, then we can find balls $B_{1}^{\prime} \subset B_{1}$ and $B_{2}^{\prime} \subset B_{2}$ which do not intersect $F$ and which have radii $\geq t C_{F}^{-1}$. (Indeed, since $F$ is regular of dimenson $d$, one can compute that the maximal number of balls of radius $t C_{F}^{-1}$ which are contained in $B_{1}$ (or $B_{2}$ ) and have disjoint doubles is about $C^{-1} C_{3}$ times the maximal number of such balls which also intersect $F$.) We want to use these balls to contradict the assumption 
that $(x, t) \in \mathcal{B}_{F}\left(C_{F}, k, M\right)$. By the usual Poincaré inequality (as in (3.1)) we have that

$$
\left|m_{B_{1}} f-m_{B_{1}^{\prime}} f\right|+\left|m_{B_{2}} f-m_{B_{2}^{\prime}} f\right| \leq C t^{-d} \int_{B(x, t) \backslash E}|\nabla f|,
$$

where $C$ depends on $C_{3}$ (but we don't care). This together with (5.45) implies that the balls $B_{1}^{\prime}$ and $B_{2}^{\prime}$ do not satisfy the analogue of (3.3) with $E$ replaced by $F, C_{0}$ replaced by $C_{F}$, and $M$ replaced by a suitable $M^{\prime}$. This proves our claim.

Note that we did not use the assumption that $t \geq 10 \operatorname{dist}(x, E)$ in the proof of the preceding claim. This assumption is needed in proving that the claim implies that $\mathcal{A}_{1}$ is a Carleson set. Specifically, this assumption implies that there are plenty of points $(y, s)$ around with $y \in E \cap B\left(x, \frac{t}{5}\right)$, and this allows us to derive the Carleson set condition on $\mathcal{A}_{1}$ (as a subset of $F \times \mathbf{R}_{+}$) from the claim, a simple accounting argument (as in Sublemma 3.9), and the fact that $\mathcal{B}_{E}\left(C_{E}, 3 k, M^{\prime}\right)$ is a Carleson set (in $E \times \mathbf{R}_{+}$), by (5.41). We omit the details.

Now let us check that $\mathcal{A}_{2}$ is a Carleson set. Let $\mathcal{B}^{\prime}$ denote the set defined in (5.38), but with $M$ replaced by a larger constant $M^{\prime}$. (This constant $M^{\prime}$ will play the same role as in the preceding case, but may not be the same number.) The key observation now is that if $C_{3}$ is large enough, and if we choose $M^{\prime}$ correctly, then $\mathcal{A}_{2} \subseteq \mathcal{B}^{\prime}$. This follows from the definitions and the fact that if $B_{1}$ and $B_{2}$ are two balls of radius $\geq t C_{1}^{-1}$ contained in $B(x, t)$, then we can find two smaller balls $B_{1}^{\prime} \subset B_{1}$ and $B_{2}^{\prime} \subset B_{2}$ which do not intersect $F$ and which have radii $\geq t C_{F}^{-1}$. The precise argument is just like the one above for $\mathcal{A}_{1}$, and so we omit the details. Once we have that $\mathcal{A}_{2} \subseteq \mathcal{B}^{\prime}$ it is not hard to prove that $\mathcal{A}_{2}$ is a Carleson set using (5.39). There is a small subtlety, though, which is that in (5.39) we are restricting the range of $R$ 's allowed, while in asserting that $\mathcal{A}_{2}$ is a Carleson set we do not make any such restriction. We are saved by the restriction on the range of $t$ 's in the definition of $\mathcal{A}_{2}$, but we leave the details as an exercise. (One has to chop large things up into small things. At least one of the authors would use a Whitney decomposition of $\Omega$.) Of course there is also the point that (5.39) applies only to $X \in K$ and not $X \in F$, but that is not serious, again because of the definition of $\mathcal{A}_{2}$.

Finally, the Carleson condition on $\mathcal{A}_{3}$ is essentially trivial. We need only observe that for each $x \in K$ we have that $\int_{\mathbf{R}_{+}} \mathbf{1}_{\mathcal{A}_{3}}(x, t) \frac{d t}{t} \leq C$. (Don't forget about (5.40).) This implies that $\mathcal{A}_{3}$ is a Carleson set, and Lemma 5.44 follows.

This completes the proof of Theorem 5.4. 
Remark 5.47. - As we mentioned before, in the preceding proof we actually showed that if $\Omega$ satisfies (5.40) and (5.41), then $K$ is contained in a regular set which satisfies the $W N P C$. The assumptions (5.40) and (5.41) are pretty reasonable, but even for an arbitrary open set $\Omega$ we have the following interior regularity result.

TheOREM 5.48. - Let $\Omega$ be an open set in $\mathbf{R}^{d+1}$, let $g \in L^{\infty}(\Omega)$ and $J(\cdot, \cdot)$ be as above, and let $(u, K)$ be an irreducible minimizer for $J(\cdot, \cdot)$. Assume that $J(u, K)<\infty$ (which is automatic when $\Omega$ is bounded). For each $x \in K$ and $r<\operatorname{dist}(x, \partial \Omega)$ let $E(x, r)$ be the set $[K \cap B(x, r)] \cup \partial B(x, r) \cup P(x, r)$, where $P(x, r)$ is any d-plane which satisfies $\operatorname{dist}(x, P(x, r)) \leq 2 r$, say. Then each such $E(x, r)$ is a d-dimensional regular set which satisfies the WNPC (and hence is uniformly rectifiable) with estimates that depend only on $d, p$, and $\|g\|_{\infty}$ but not on $x$ or $r$.

This is proved in exactly the same manner as Theorem 5.4 was. We added the hyperplane $P(x, r)$ only to get a lower bound on $H^{d}(E(x, r) \cap B(y, t))$ (as in (5.43)) for large radii $t$ (so that $E(x, r)$ satisfies the definition of regularity in Section 2.). Note that we are implicitly using the simple fact that $P(x, r) \cup B(x, r)$ satisfies the WNPC with bounded constants.

Remark 5.49. - If $\partial \Omega$ is contained in a uniformly rectifiable set $\mathrm{E}$ of dimension $d$, and if $(u, K)$ is an irreducible minimizer as above, then $E \cup K$ is uniformly rectifiable (and hence $K$ is contained in a uniformly rectifiable set). To see this it is more convenient to use the characterization of uniform rectifiability in terms of the existence of "big pieces of bilipschitz images of $\mathbf{R}^{d}$ ". This condition is defined in Definition 1.33 on p. 15 of [DS3], and its equivalence with Definition 2.9 is stated in Theorem 1.57 on p. 22 of [DS3]. (See also the main result of [DS1].) Assuming that $E \supseteq \partial \Omega$ is uniformly rectifiable, let us check that $F=K \cup E$ is too. It is easy to see that $F$ is regular, as in the discussion around (5.42) and (5.43). Let $x \in F$ and $r>0$ be given, and let us check that $F \cap B(x, r)$ has a big piece of a bilipschitz image of $\mathbf{R}^{d}$. When $x \in K$ and $r<\operatorname{dist}(x, \partial \Omega)$ there is a big piece of a bilipschitz image of $\mathbf{R}^{d}$ inside $K \cap B(x, r)$ because of Theorem 5.48. (It is helpful to take $P(x, r)$ to be disjoint from $B(x, r)$ here, to keep it from interfering with $K$ in $B(x, r)$.) If $x \in K$ and $r \leq 10 \operatorname{dist}(x, \partial \Omega)$, then we can also get a big piece of a bilipschitz image of $\mathbf{R}^{d}$ inside $K \cap B(x, r)$ by applying the preceding case to $\frac{r}{10}$. In the remaining cases we have either $x \in K$ and $r>10 \operatorname{dist}(x, \partial \Omega)$ or else $x \in E$. In either case there must be a big piece of a bilipschitz image of $\mathbf{R}^{d}$ inside $E \cap B(x, r)$, since $E$ is uniformly rectifiable. Thus $F$ is uniformly rectifiable, as desired. 
(See Section 7 of [DS4] for a slightly more detailed treatment of the same point in the simpler $d=1$ situation.)

\section{SETS OF PRESCRIBED MEAN CURVATURE}

Let $\Omega$ be an open set in $\mathbf{R}^{d+1}, d \geq 1$, and let $H \in L^{1}(\Omega)$ be given. If $X$ is a measurable subset of $\Omega$, define $\mathcal{F}(X)$ by

$$
\mathcal{F}(X)=|\partial X|(\Omega)+\int_{\Omega} \mathbf{1}_{X}(x) H(x) d x
$$

where $|\partial X|(\Omega)$ denotes the "perimeter" of $X$ in $\Omega$, i.e.,

$$
=\sup \left\{\int \mathbf{1}_{X}(x) \operatorname{div} G(x) d x: G \in C_{0}^{1}\left(\Omega, \mathbf{R}^{d+1}\right),\|G\|_{\infty} \leq 1\right\} .
$$

Here $C_{0}^{1}\left(\Omega, \mathbf{R}^{d+1}\right)$ means $\mathbf{R}^{d+1}$-valued $C^{1}$ mappings with compact support in $\Omega$. In other words, $|\partial X|(\Omega)$ is the total mass of the distributional gradient of the characteristic function of $X$. If $X$ is a local minimizer for $\mathcal{F}(\cdot)$, so that $\mathcal{F}(X) \leq \mathcal{F}(Y)$ whenever $(X \backslash Y) \cup(Y \backslash X)$ is contained in a compact subset of $\Omega$, then $X$ is said to have generalized mean curvature $H$. Indeed, if this condition holds, and if $X$ and $H$ are sufficiently well behaved, then the classical mean curvature of $X$ is given by (a constant multiple of) the restriction of $H$ to the boundary of $X$. See [GMT] and the references therein for more information about this notion of generalized mean curvature.

Note that in general the restriction of $H$ to the boundary of $X$ does not make sense, because $H$ is merely integrable, and that the values of $H$ off $X$ are not at all determined by $X$. Also, when $H \equiv 0$ this variational problem reduces to the usual least area problem of minimal surfaces.

If $X$ has generalized mean curvature $H$, then what can we say about the structure of the boundary of $X$ in terms of the behavior of $H$ ? In general we have to be careful and work with the "reduced boundary" of $X$ (as in [Gi]), rather than the topological boundary. (This is not a surprise, since $X$ is really defined only up to sets of Lebesgue measure 0 .) Since our competitors have finite perimeter their reduced boundaries are rectifiable. (Again, see [Gi].) Under suitable conditions on the size of $H$ (e.g., $H \in L^{p}$, $p>d+1$ ) one can get regularity theorems analogous to those for minimal surfaces in codimension 1. (See the introduction and references in [GMT].) 
Since we have rectifiability of the reduced boundary for any integrable function $H$, we can wonder whether we can get uniform rectifiability under uniform integrability conditions on $H$. Because of the scaling in this problem the natural "uniform integrability" condition to impose on $H$ is

$$
\int_{B(x, r)}|H(y)| d y \leq M r^{d}
$$

for some constant $M$ and all $x \in \mathbf{R}^{d+1}$ and $r>0$. (We may as well take $H$ to be 0 outside $\Omega$.) Note that this is automatically true when $H \in L^{d+1}$, by Hölder's inequality, and in that case one can even take $M$ to be small if one restricts one's attention to sufficiently small radii $r$.

It turns out that (6.3) is really too weak to obtain anything like uniform rectifiability of the boundary of $X$. In order to build examples we shall use the following result (given as Lemma 1.3 in [GMT]).

Lemma 6.4. - Suppose that $X$ is a set with smooth boundary in $\Omega$, and assume that the outer normal unit vector $\nu(x)$ at $x \in \Omega \cap X$ can be extended to a vector field $V: \Omega \rightarrow \mathbf{R}^{d+1}$ with $V \in W^{1,1}(\Omega) \cap C(\Omega),\|V\|_{\infty} \leq 1$. If

$$
H(x)=-\operatorname{div} V(x)
$$

then $X$ has generalized mean curvature $H$.

This lemma is useful because it permits us to build complicated sets with generalized mean curvature $H$, with $H$ under some control, by taking many nice little blobs (like balls) and scattering them about. To make this precise we begin with a trivial observation.

LEMMA 6.6. - If $B$ is a ball of radius $r$ in $\mathbf{R}^{d+1}$, then there is a smooth vector field $V: \Omega \rightarrow \mathbf{R}^{d+1}$ supported in $2 B$ such that $\|V\|_{\infty} \leq 1$, $\|\nabla V\|_{\infty} \leq C r^{-1}$, and $V$ agrees with the outer unit normal of $\partial B$ on $\partial B$.

Thus given a ball $B$ we can find an $H$ which works and which is supported in $2 B$. To build complicated sets we need only arrange some balls in a suitable way. For simplicity let us give examples only in $\mathbf{R}^{2}$, although the construction can be adapted easily to higher dimensions.

Let $S$ denote the unit square $[0,1] \times[0,1]$ in $\mathbf{R}^{2}$. Let $T$ denote the union of the four squares with sidelength $\frac{1}{4}$ in the corners of $S$. Given a set $A$ in $\mathbf{R}^{2}$ which is a finite disjoint union of squares, let us call a set $A^{\prime}$ an admissible modification of $A$ if we can obtain $A^{\prime}$ from $A$ by taking one of the squares in $A$ and replacing it with the four smaller corner squares of size $\frac{1}{4}$ the size of the square which is being replaced. Thus 
$S$ has only one admissible modification, which is $T$, while $T$ has four admissible modifications. Let $\mathcal{C}$ denote the collection of sets in the plane consisting of $S$, its modification $T$, all the modifications of $T$, and the modifications of the modifications, etc. Thus every element of $\mathcal{C}$ is a finite union of squares, each with sides parallel to the axes and with sidelength some negative power of 4 . Let $\widehat{\mathcal{C}}$ denote the collection of sets consisting of the elements of $\mathcal{C}$ and all sets obtained by taking a set in $\mathcal{C}$ and deleting any number of its constituent squares.

In short, $\mathcal{C}$ and $\widehat{\mathcal{C}}$ are collections of subsets of the plane which are finite unions of squares arranged in a Cantor-like way. If one is exhaustive about replacing all the squares by smaller squares, then one obtains the usual approximations to a true Cantor set. The difference between $\mathcal{C}$ and $\widehat{\mathcal{C}}$ is that elements of $\mathcal{C}$ are never too sparse, while elements of $\widehat{\mathcal{C}}$ can be very sparse (by deleting many little squares).

Now suppose that $Y$ is an element of $\widehat{\mathcal{C}}$, so that $Y$ is the union of some squares $\left\{\sigma_{i}\right\}_{i}$. Let $\left\{B_{i}\right\}_{i}$ be the collection of open balls such that $2 B_{i}$ is the largest possible disk inside $\sigma_{i}$. Set $X=\bigcup B_{i}$. Let $V$ be the vector field on $\mathbf{R}^{2}$ supported on $Y$ which is obtained by applying Lemma 6.6 to each of the $B_{i}$ 's and adding the results. If we take $H$ to be as in (6.5), then we conclude from Lemma 6.4 that $X$ has generalized mean curvature $H$.

LEMMA 6.7. - Under the notations and conditions of the preceding paragraph, there is a constant $M$ which does not depend on the choice of $Y \in \widehat{\mathcal{C}}$ such that $H$ satisfies (6.3).

This is not hard to check. From Lemma 6.6 we have that $|H| \leq$ $C \operatorname{diam}\left(\sigma_{i}\right)^{-1}$ for each $\sigma_{i}$. The estimate (6.3) follows from the fact that in the construction of elements of $\widehat{\mathcal{C}}$ we are always careful to replace a square by the union of four smaller squares whose total sidelength is the same as that of the original square. We omit the details.

Although each $X$ arising in this manner is just a finite union of disks, they are not uniformly well-behaved. For the purposes of this discussion let us agree to call a compact set (Ahlfors) regular if it satisfies the same definition as given in the beginning of Section 2 except that we ask only that (2.1) hold for $r$ less than the diameter of the set. It is not true then that $\partial X$ is regular (of dimension 1) with a uniformly bounded constant as $Y$ runs through $\widehat{\mathcal{C}}$, since we can make $Y$ and hence $\partial X$ as thin as we want, by the definition of $\widehat{\mathcal{C}}$. This is not the case for $X$ 's which result from a $Y \in \mathcal{C}$; for these $X$ 's $\partial X$ is regular with a uniformly bounded constant. (This is not hard to check from the definition of $\mathcal{C}$. It comes down to the fact that when we replace a square by the four corner squares of one-fourth the size, we do not reduce the length of the boundary.) However, even for this 
more restricted class of sets $\partial X$ we do not get uniform rectifiability with uniform bounds. To see this, let $K$ be the Cantor set obtained by starting with the unit square $S$ by $T$ above, then replacing each of the four squares in $T$ by a smaller copy of $T$, etc., so that in the limit we get the totally disconnected compact set $K . K$ has positive and finite one-dimensional Hausdorff measure, but it is well-known that it cannot be realized as a subset of a curve (or even a connected set) of finite length. (In fact, its intersection with any such curve must have length 0 .) If the $\partial X$ 's for $Y \in \mathcal{C}$ were uniformly rectifiable with uniform bounds, then we would be able to conclude that $K$ is contained in a rectifiable curve, because we can approximate $K$ by $\partial X$ 's in the Hausdorff metric.

Thus we conclude that (6.3) is not strong enough to imply anything like regularity or uniform rectifiability of $\partial X$. We shall spare the reader the technicalities of producing $X$ 's such that $\partial X$ is not regular, or such that $\partial X$ is regular but not uniformly rectifiable, rather than simply nice $\partial X$ 's as above for which the regularity or uniform rectifiability constants are arbitrarily large.

Of course the situation would be different if we were to assume that (6.3) holds with a small constant.

Remark 6.8. - There are other ways of seeing that (6.3) itself has no hope of implying anything like uniform rectifiability which are more complicated but which make clearer the principles involved. There is a nice theorem of Varopolous [V2] which implies that any $L^{\infty}$ function $f$ on $\mathbf{R}^{d}$ has an extension $F$ to $\mathbf{R}^{d+1}$ such that $|\nabla F|$ satisfies (6.3). It turns out that there is nothing really special about a hyperplane here, in the sense that the same result would work for much more general sets in $\mathbf{R}^{d+1}$ (like regular sets of dimension $d$ ). Modulo a priori smoothness assumptions, then, the requirement that the unit normal on the boundary of a domain have an extension $V$ which satisfies the conclusions of Lemma 6.4, with $H$ also satisfying (6.3), does not really put much of a restriction on this unit normal vector, since it lies in $L^{\infty}$ anyway. Our Cantor set examples made this precise and explicit, but the truth of the matter is that in this context (6.3) is not much of a condition.

If, on the other hand, our extension $F$ of $f$ has the property that $|\nabla F|$ satisfies (6.3) with small constant, then Varopolous [V1] showed that $f$ must have small BMO norm (and conversely). Roughly speaking this means that the average oscillations of $f$ are small, but we shall spare the reader the precise definitions. This type of condition on the unit normal of a domain is pretty strong. (See [Se3].) It is also stronger than we would have here 
when the constant in (6.3) is small, because it would correspond to a condition on all the (generalized) principle curvatures, instead of only the generalized mean curvature.

\section{REFERENCES}

[Am] L. Ambrosio, Existence theory for a new class of variational problems, Arch. Rational Mech. Anal., Vol. 111, 1990, pp. 291-322.

[CL1] M. CARRIERo and A. LEACI, Existence theorem for a Dirichlet problem with free discontinuity set, Nonlinear Analysis, Theory, Methods \& Applications, Vol. 15, 1990, pp. 661-677.

[CL2] M. CARRIERo and A. LEACI, $\mathbf{S}^{k}$-Valued Maps minimizing the $L^{p}$ norm of the gradient with free discontinuities, Ann. Sc. Norm. Sup. Pisa, Vol. 18, 1991, pp. 321-352.

[DCL] E. De Giorgi, M. Carriero and A. Leaci, Existence theorem for a minimum problem with free discontinuity set, Arch. Rational Mech. Anal., Vol. 108, 1989, pp. 195-218.

[Da] G. DAVID, Morceaux de graphes lipschitziens et intégrales singulières sur une surface, Revista Matematica Iberoamericana, Vol. 4, 1, 1988, pp. 73-114.

[DJ] G. DAVID and D. JERISON, Lipschitz approximations to hypersurfaces, harmonic measure, and singular integrals, Indiana U. Math. Journal., Vol. 39, 3, 1990, pp. 831-845.

[DMS] G. Dal Maso, J.-M. Morel and S. SoliminI, A variational method in image segmentation: Existence and approximation results, Acta Math., Vol. 168, 1992, pp. 89-151.

[DS1] G. DAVID and S. SEMmES, Singular Integrals and Rectifiable Sets in $\mathbf{R}^{n}$ : au-delà des graphes lipschitziens, Astérisque 193, Société Mathématique de France, 1991.

[DS2] G. DAVID and S. SEMmES, Quantitative rectifiability and Lipschitz mappings, Transactions A.M.S., Vol. 337, 1993, pp. 855-889.

[DS3] G. DAVID and S. SEMmes, Analysis of and on Uniformly Rectifiable Sets, Mathematical surveys and monographs, Vol. 38, 1993, American Mathematical Society.

[DS4] G. DAVID and S. Semmes, On the singular sets of minimizers of the Mumford-Shah functional, to appear J. Math. Pures Appl.

[DS5] G. DaVID and S. SEMmes, On a variational problem from image processing, Proceedings of the conference in Honor of Jean-Pierre Kahane, Journ. of Fourier Analysis and Applications, 1995, pp. 161-187.

[Fa] K. FALCONER, The geometry of fractal sets, Cambridge University Press, 1984.

[Fe] H. FEDERER, Geometric Measure Theory, Grundlehren der Mathematishen Wissenschaften 153, Springer-Verlag 1963.

[Ga] J. Garnetr, Bounded Analytic Functions, Academic Press, 1981.

[Gi] E. Giusti, Minimal Surfaces and Functions of Bounded Variation, Birkhäuser, 1984.

[GMT] H. Gonzales, U. Massari and I. Tamanin, Boundaries of Prescribed Mean Curvature, Rend. Mat. Acc. Lincei s. 9, Vol. 4, 1993, pp. 197-206.

[Hr] T. HRYCAK, Ph.D. thesis, Yale university.

[Je] J. L. JouRNÉ, Calderón-Zygmund Operators, Pseudodifferential Operators, and the Cauchy Integral of Calderón, Lecture Notes. in Math., Vol. 994, 1983, Springer-Verlag.

[Js] P. JoNES, Lipschitz and bi-Lipschitz functions, Rev. Mat. Iberoamericana, Vol. 4 , 1988, pp. 115-122.

[Ma] P. Matrila, Geometry of Sets and Measures in Euclidean Spaces, to appear, Cambridge Studies in Advanced Math., Cambridge Univ. Press. 
[MoS] J.-M. MoRel and S. Solimini, Variational Methods in Image Segmentation, Progress in Nonlinear differential equations and their applications, Vol. 14, Birkhauser, 1995.

[MuS] D. MUMFORD and J. SHAH, Optimal approximations by piecewise smooth functions and associated variational problems, Comm. Pure Appl. Math., Vol. 42, 1989, pp. 577-685.

[Se1] S. SEMmES, A criterion for the boundedness of singular integrals on hypersurfaces, Trans. Amer. Math. Soc., Vol. 311, 2, 1989, pp. 501-513.

[Se2] S. SEMmes, Analysis vs. geometry on a class of rectifiable hypersurfaces in $\mathbf{R}^{n}$, Indiana Math. J., Vol. 39, 1990, pp. 1005-1035.

[Se3] S. Semmes, Hypersurfaces in $\mathbf{R}^{n}$ whose unit normal has small BMO norm, Proc. Amer. Math. Soc., Vol. 112, 1991, pp. 403-412.

[St] E. M. STEIN, Singular integrals and differentiability properties of functions, Princeton University Press 1970.

[V1] N. Varopoulos, BMO functions and the $\bar{\partial}$ equation, Pacific J. Math., Vol. 71, 1977, pp. 221-273.

[V2] N. VAROPOULOS, A remark on BMO and bounded harmonic functions, Pacific J. Math., Vol. 73, 1977, pp. 257-259.

(Manuscript received October 24, 1994.) 